\title{
Evaluation of organic markers for chemical mass balance source apportionment at the Fresno Supersite
}

\author{
J. C. Chow ${ }^{1}$, J. G. Watson ${ }^{1}$, D. H. Lowenthal ${ }^{1}$, L. W. A. Chen ${ }^{1}$, B. Zielinska ${ }^{1}$, L. R. Mazzoleni ${ }^{2}$, and K. L. Magliano ${ }^{3}$ \\ ${ }^{1}$ Desert Research Institute, Reno, NV, USA \\ ${ }^{2}$ Colorado State University, Fort Collins, CO, USA \\ ${ }^{3}$ California Air Resources Board, Sacramento, CA, USA
}

Received: 23 August 2006 - Published in Atmos. Chem. Phys. Discuss.: 17 October 2006

Revised: 31 January 2007 - Accepted: 8 February 2007 - Published: 10 April 2007

\begin{abstract}
Sources of $\mathrm{PM}_{2.5}$ at the Fresno Supersite during high $\mathrm{PM}_{2.5}$ episodes occurring from 15 December 2000-3 February 2001 were estimated with the Chemical Mass Balance (CMB) receptor model. The ability of source profiles with organic markers to distinguish motor vehicle, residential wood combustion (RWC), and cooking emissions was evaluated with simulated data. Organics improved the distinction between gasoline and diesel vehicle emissions and allowed a more precise estimate of the cooking source contribution. Sensitivity tests using average ambient concentrations showed that the gasoline vehicle contribution was not resolved without organics. Organics were not required to estimate hardwood contributions. The most important RWC marker was the water-soluble potassium ion. The estimated cooking contribution did not depend on cholesterol because its concentrations were below the detection limit in most samples. Winter time source contributions were estimated by applying the CMB model to individual and average sample concentrations. RWC was the largest source, contributing 29-31\% of measured $\mathrm{PM}_{2.5}$. Hardwood and softwood combustion accounted for $16-17 \%$ and $12-15 \%$, respectively. Secondary ammonium nitrate and motor vehicle emissions accounted for $31-33 \%$ and $9-15 \%$, respectively. The gasoline vehicle contribution (3-10\%) was comparable to the diesel vehicle contribution (5-6\%). The cooking contribution was 5-19\% of $\mathrm{PM}_{2.5}$. Fresno source apportionment results were consistent with those estimated in previous studies.
\end{abstract}

\section{Introduction}

According to the California emission inventory, area-wide sources account for about $76 \%$ of the statewide emissions

Correspondence to: J. C. Chow

(judy.chow@dri.edu) of directly emitted $\mathrm{PM}_{2.5}$ (582 out of 765 tons/day [t/day]) (California Air Resources Board, 2004). Approximately half of the remaining directly emitted $\mathrm{PM}_{2.5}(13 \%)$ originates from on-road and off-road vehicle emissions ( $97 \mathrm{t} /$ day). Area sources include road/fugitive dust (248t/day), residential and agriculture burning ( $123 \mathrm{t} /$ day), construction ( $42 \mathrm{t} /$ day), and cooking (19t/day). These contributions vary spatially and temporally (Chow et al., 2006a; Rinehart et al., 2006). For example, residential wood combustion (RWC) is common in populated urban areas during winter.

Previous San Joaquin Valley (SJV) source apportionment studies have shown the importance of fugitive dust, vehicle exhaust, agricultural burning and RWC, and cooking contributions to $\mathrm{PM}_{2.5}$ and $\mathrm{PM}_{10}$ (Chow et al., 1992; Magliano et al., 1999; Schauer and Cass, 2000). Primary $\mathrm{PM}_{2.5}$ and $\mathrm{PM}_{10}$ contributions from industrial sources were negligible. Chow et al. (1992) and Magliano et al. (1999) used Chemical Mass Balance (CMB) modeling with elements, inorganic ions, organic carbon (OC), and elemental carbon (EC). Neither of these studies distinguished diesel- from gasoline-powered motor vehicle contributions or vegetative burning from cooking contributions. Both applications included a "pure" OC profile to explain ambient OC concentrations. Magliano et al. (1999) suggested that the pure OC source represented unidentified activities that might also include secondary organic aerosol (SOA).

Organic compounds measured by different methods have been used to help distinguish among source contributions to the PM carbon fraction (Schauer et al., 1996; Watson et al., 1998a; Zheng et al., 2002, 2006; Manchester-Neesvig et al., 2003; Hannigan et al., 2005; Labban et al., 2006). Schauer et al. (2000) applied the CMB model to three multiday episodes during winter 1995/1996 and reported contributions from diesel and gasoline exhaust, hardwood and softwood combustion, cooking, and natural gas combustion at four SJV locations, including the Fresno Supersite (Watson et al., 2000), where $\mathrm{PM}_{2.5}$ carbon levels are high during

Published by Copernicus GmbH on behalf of the European Geosciences Union. 
winter (Chow and Watson, 2002; Chow et al., 2006a, b; Park et al., 2006).

Results are reported here from CMB source apportionment of samples at the Fresno Supersite during high $\mathrm{PM}_{2.5}$ episodes in winter 2000/2001 as part of the California Regional $\mathrm{PM}_{10} / \mathrm{PM}_{2.5}$ Air Quality Study (CRPAQS; Watson and Chow, 2002; Chow et al., 2005a; Rinehart et al., 2006). These data are used with source profile measurements to quantify and evaluate the uncertainty of source contributions during this period using the effective variance solution (Watson et al., 1984) to the CMB equations. Tests with simulated data and with and without the inclusion of marker compounds were undertaken to determine the feasibility and stability of the source contribution estimates.

\section{Methods}

\subsection{Ambient measurements}

Sampling and analysis details are reported elsewhere (Chow, 1995; Chow et al., 2005a, b) and summarized here. The Fresno Supersite is located at 3425 First Street, Fresno, CA, approximately five $\mathrm{km}$ from the downtown district. Air quality monitors are operated on the roof of a two-story building. Samples were collected with Desert Research Institute (DRI; Reno, NV) sequential filter samplers (SFS) preceded by $\mathrm{PM}_{2.5}$ size-selective inlets (Sensidyne Bendix 240 cyclones) and aluminum oxide tubular nitric acid $\left(\mathrm{HNO}_{3}\right)$ denuders (Chow et al., 2005b). Teflon-membrane (Pall Sciences, R2PJ047, Ann Arbor, MI) filters were analyzed for $\mathrm{PM}_{2.5}$ mass by gravimetry and for elements by x-ray fluorescence (Watson et al., 1999). Quartz-fiber (Pall Sciences, QAT2500-VP, Ann Arbor, MI) filters were analyzed for chloride $\left(\mathrm{Cl}^{-}\right)$, nitrate $\left(\mathrm{NO}_{3}^{-}\right)$, and sulfate $\left(\mathrm{SO}_{4}^{-}\right)$by ion chromatography (Chow and Watson, 1999), ammonium $\left(\mathrm{NH}_{4}^{+}\right)$ by automated colorimetry, and water-soluble sodium $\left(\mathrm{Na}^{+}\right)$ and potassium $\left(\mathrm{K}^{+}\right)$by atomic absorption spectrometry. OC and EC were analyzed by the IMPROVE thermal/optical reflectance (TOR) protocol (Chow et al., 1993, 2001, 2004a, 2005c). OC1-OC4 fractions evolve at 120, 250, 450, and $550^{\circ} \mathrm{C}$, respectively, in a $100 \%$ helium $(\mathrm{He})$ atmosphere. The OP fraction is pyrolyzed OC. OC is the sum of OC1-OC4 plus OP. The EC1-EC3 fractions evolve at 550, 700, and $800^{\circ} \mathrm{C}$, respectively, in a $98 \% \mathrm{He} / 2 \%$ oxygen $\left(\mathrm{O}_{2}\right)$ atmosphere. EC is the sum of EC1-EC3 minus OP.

$\mathrm{PM}_{2.5}$ samples for semi-volatile organic compounds (SVOCs) were acquired with DRI sequential fine particle/semi-volatile organic samplers on Teflonimpregnated glass-fiber filters (TIGF) to collect particles followed by PUF/XAD/PUF (polyurethane foam, polystyrene-divinylbenzene XAD-4 resin) cartridges (Zielinska et al., 1998, 2003). Two- to four-ring polycyclic aromatic hydrocarbons (PAHs), methoxy-phenol derivatives, alkanes, and organic acids are present in both the gas and particle phases while hopanes, steranes, and high molecular weight organic acids and alkanes are present mainly in the particle phase (Zielinska et al., 2004a). For SVOC analysis (Zielinska and Fujita, 2003; Zielinska et al., 2003; Rinehart, 2005; Rinehart et al., 2006), deuterated internal standards were added to each filter-sorbent pair. TIGF/XAD and PUF samples were extracted in dichloromethane and $10 \%$ diethyl ether in hexane, respectively, followed by acetone extraction using an Accelerated Solvent Extractor (ASE-300, Dionex, Sunnyvale, CA). The solvent volumes were generally $150 \mathrm{ml}$. The solvent extracts from the PUF plugs and filter-XAD pairs for individual samples were combined and concentrated by rotary evaporation at $20^{\circ} \mathrm{C}$ under gentle vacuum to $\sim 1 \mathrm{ml}$. The samples were then split into two equivalent fractions. The final sample volume of both halves was reduced under a gentle stream of nitrogen and adjusted to $0.1 \mathrm{ml}$ with acetonitrile.

The non-derivatized SVOC fraction was analyzed by electron impact (EI) gas chromatography/mass spectrometry (GC/MS) for PAHs, hopanes, steranes, and high molecular weight alkanes on a Varian CP 3800 GC with a CP-Sil 8 Chrompack (Varian, Inc., Palo Alto, CA) column connected to a Varian Saturn 2000 Ion Trap. Polar compounds in the second fraction (organic acids, cholesterol, sitosterol, levoglucosan, and methoxy-phenols) were converted to their trimethylsilyl derivatives using a mixture of N,O-bis (trimethylsilyl) trifluoroacetamide with $1 \%$ trimethylchlorosilane, and pyridine. The calibration solutions were freshly prepared and derivatized just prior to the analysis of each sample set and all samples were analyzed by GC/MS within $18 \mathrm{~h}$ to avoid degradation. Samples were analyzed by chemical ionization GC/MS with isobutane as a reagent gas using a Varian CP 3800 GC with a CP-Sil 8 Chrompack (Varian, Inc.) column connected to a Varian Saturn 2000 Ion Trap (Zielinska et al., 2003; Rinehart, 2005, Rinehart et al., 2006).

Samples were collected from 15 through 18 December 2000, from 26 through 28 December 2000, from 4 through 7 January 2001, and from 31 January through 3 February 2001 based on forecasts of high $\mathrm{PM}_{2.5}$ conditions. Forecasting was done by San Joaquin Valley Air Pollution Control District meteorologists using a regression-based prognostic model that predicts 5-day $\mathrm{PM}_{10}$ and $\mathrm{PM}_{2.5}$ concentrations based on variables including atmospheric stability, wind speed, upper-air temperature, and continuous $\mathrm{NO}_{3}^{-}$ and carbon measurements. The study management team reviewed the model predictions daily over an afternoon conference call, and initiated intensive operating periods when the expected $\mathrm{PM}_{2.5}$ concentrations exceeded the national $\mathrm{PM}_{2.5}$ standard of $65 \mu \mathrm{g} / \mathrm{m}^{3}$. Samples were taken throughout the day to bound periods of differing source contributions (Watson and Chow, 2002; Chow et al., 2006a; Watson et al., 2006a, b): 1) 00:00-05:00 PST (Pacific Standard Time, GMT-8) for an aged nighttime mixture, 2) 05:00-10:00 PST for the morning rush-hour, 3) 10:00-16:00 PST for mixing 
down of aged/secondary aerosol; and 4) 16:00-24:00 PST for evening traffic, cooking, and home heating.

\subsection{Chemical Mass Balance model}

The CMB receptor model (Hidy and Friedlander, 1971) describes $C_{i t}$, the ambient concentration of the $i$-th chemical species measured at time $t$, as the linear sum of contributions from $J$ sources:

$C_{i t}=\sum_{i=1}^{J} F_{i j} S_{j t}+E_{i t}$

where $F_{i j}$ is the fractional abundance (source profile) of the $i$-th species in the $j$-th source type, $S_{j t}$ is the mass contribution of the $j$-th source at time $t$, and $E_{i t}$ represents the difference between the measured and estimated ambient concentration. Ideally, $E_{i t}$ reflects random measurement uncertainty. There are numerous solutions to the CMB equations, including Positive Matrix Factorization (PMF) and UNMIX (Watson et al., 2002a; Watson and Chow, 2004), which have also been applied to $\mathrm{PM}_{2.5}$ data in central California (Chen et al., 2007). The effective variance weighted least squares minimization solution (Watson et al., 1984) is most commonly used for obtaining source contribution estimates $\left(S_{j t}\right)$, as implemented with CMB8 software (Watson et al., 1997, 1998b). As applied here, samples with $S_{j t}<0$ are eliminated and the solution is iterated until all remaining $S_{j t}$ are positive for each sample. Wang and Hopke (1989) showed that this approach provides more precise estimates than does an unconstrained solution for sources whose profiles are collinear.

$\mathrm{CMB}$ results are evaluated with performance measures such as r-square (R SQR) and chi-square (CHI SQR) and the percentage of measured mass (PCMASS) accounted for by the sum of the $S_{j t}$ (Watson and Chow, 2005). Although acceptable values for these metrics are necessary, they are not sufficient to guarantee $S_{j t}$ that represent reality. The most important potential biases in the CMB model are related to improper specification of the contributing sources and unrealistic source profiles.

\subsection{Source profiles}

The $\mathrm{PM}_{2.5}$ source profiles in Table 1 were derived from emission studies of vehicle exhaust, wood burning, and cooking specific to fuels and operating conditions in California. Owing to differences in methods used to measure thermal carbon fractions (Watson et al., 2005), it is necessary to use profiles that were obtained using the same method applied to the receptor samples. It is also important that the organic compounds measured in the source profiles match those measured at the receptor. These profiles have been integrated into a documented data base with other recent profiles that is available from the authors (Chow et al., 2005a) and are being incorporated into the U.S. EPA's SPECIATE data base (U.S. EPA, 2007).
Composite diesel (DIES) and gasoline (GAS) exhaust profiles were derived from many dynamometer tests on a wide range of vehicles during the summer of 2001 (Fujita et al., $2006,2007^{1}$ ). The sum of species in the diesel exhaust profile was larger than the measured mass, probably because the Teflon filters on which mass was determined were overloaded or because of VOC absorption by the quartz-fiber filter (Turpin et al., 1994). Therefore, the diesel exhaust profile (DIES) was normalized to the sum of species. The most useful components for separating diesel- from gasoline-exhaust contributions are three PAHs (i.e., indeno[123-cd]pyrene, benzo(ghi)perylene, and coronene) and EC (Miguel et al., 1998; Zielinska et al., 2004a, b; Fujita et al., 2007 ${ }^{1}$ ). High temperature $\mathrm{EC}\left(\mathrm{EC} 2\right.$, evolved at $700^{\circ} \mathrm{C}$ in an oxidative environment; Watson et al., 1994) was abundant in the diesel engine tests.

Hardwood (BURN-H) and softwood (BURN-S) profiles from RWC were determined from oak, eucalyptus, and almond (hardwood) and tamarack (softwood) burns under controlled conditions (McDonald et al., 2000; Fitz et al., 2003). The emission inventory suggested that there was more hardwood than softwood combustion in Fresno during 1995 (Magliano et al., 1999). $\mathrm{PM}_{2.5} \mathrm{~K}^{+}$and polar organic compounds including levoglucosan, syringols, and guaiacols are markers for wood burning emissions (Rinehart, 2005; Rinehart et al., 2006).

Meat cooking (McDonald et al., 2003; Chow et al., 2004b) is represented by composite meat cooking profiles for charbroiled chicken (CHCHICK), chicken over propane (PRCHICK), and charbroiled hamburger (CHHAMB); an average meat cooking profile (COOK) was derived from these three. A smoked chicken profile (SMCHICK) was not included because it was enriched in levoglocosan from wood smoke. The primary markers for cooking are thought to be polar compounds such as cholesterol, palmitic acid, palmitoleic acid, stearic acid, and oleic acid (Fraser et al., 2003; Rinehart, 2005; Rinehart et al., 2006). However, these fatty acids can be emitted by sources other than meat cooking as they are abundant in seed oils used for cooking processes. Fatty acids are also present in vegetative burning, personal care products, plastic additives, household and industrial cleaners, and other domestic products. Cholesterol, a marker compound for meat cooking (Rogge et al., 1991), is also a constituent of biogenic detritus (Simoneit, 1989).

Geological source profiles were determined from SJV suspended dust samples (Ashbaugh et al., 2003; Chow et al., 2003) representing a wide range of urban and non-urban soils. Composite source profiles were created for: paved road dust (PVRD), unpaved road dust (UPVRD), agricultural soil

\footnotetext{
${ }^{1}$ Fujita, E. M., Campbell, D. E., Arnott, W. P., Zielinska, B., and Chow, J. C.: Evaluations of source apportionment methods for determining contributions of gasoline and diesel exhaust to ambient carbonaceous aerosols, J. Air Waste Manage. Assoc., in review, 2007.
} 
Table 1. Source profiles (percent of emitted $\mathrm{PM}_{2.5}$ ) used in CMB modeling for Fresno samples acquired during the CRPAQS winter intensive study.

\begin{tabular}{|c|c|c|c|c|c|c|c|}
\hline \multirow[b]{2}{*}{ Chemical Species } & \multirow[b]{2}{*}{$\begin{array}{l}\text { Species } \\
\text { Abbreviation }\end{array}$} & \multicolumn{6}{|c|}{ Source Type and Code } \\
\hline & & $\begin{array}{l}\text { Paved Road } \\
\text { PVRD }\end{array}$ & $\begin{array}{l}\text { Gasoline Vehicle } \\
\text { GAS }\end{array}$ & $\begin{array}{l}\text { Diesel Vehicle } \\
\text { DIES }\end{array}$ & $\begin{array}{l}\text { Hardwood Combustion } \\
\text { BURN-H }\end{array}$ & $\begin{array}{l}\text { Softwood Combustion } \\
\text { BURN-S }\end{array}$ & $\begin{array}{l}\text { Smoked Chicken } \\
\text { SMCHICK }\end{array}$ \\
\hline Chloride & $\mathrm{Cl}^{-}$ & $0.1027 \pm 0.1839$ & $0.4769 \pm 0.4318$ & $0.2371 \pm 0.3495$ & $1.4719 \pm 1.8146$ & $0.1061 \pm 0.0323$ & $1.2589 \pm 0.7814$ \\
\hline Nitrate & $\mathrm{NO}_{3}^{-}$ & $0.0435 \pm 0.1817$ & $1.6545 \pm 1.2115$ & $0.1351 \pm 0.3835$ & $0.6803 \pm 0.0567$ & $0.1534 \pm 0.0293$ & $0.4196 \pm 0.1199$ \\
\hline Sulfate & $\mathrm{SO}_{4}^{\equiv}$ & $0.2787 \pm 0.1881$ & $6.7749 \pm 6.9651$ & $3.5862 \pm 2.9797$ & $1.4179 \pm 0.6204$ & $0.5794 \pm 0.0597$ & $0.4235 \pm 0.2699$ \\
\hline Ammonium & $\mathrm{NH}_{4}^{+}$ & $0.3233 \pm 0.2305$ & $3.0173 \pm 3.1377$ & $1.1804 \pm 1.1875$ & $0.4565 \pm 0.3963$ & $0.2122 \pm 0.0312$ & $0.1407 \pm 0.1188$ \\
\hline Water-Soluble sodium & $\mathrm{Na}^{+}$ & $0.0789 \pm 0.0351$ & $0.0000 \pm 0.0010$ & $0.0000 \pm 0.0010$ & $0.3045 \pm 0.0252$ & $0.1544 \pm 0.0117$ & $0.2170 \pm 0.0291$ \\
\hline Water-Soluble Potassium & $\mathrm{K}^{+}$ & $0.1509 \pm 0.0899$ & $0.0699 \pm 0.0682$ & $0.1552 \pm 0.0529$ & $2.9389 \pm 0.3286$ & $0.8124 \pm 0.0594$ & $0.3454 \pm 0.0354$ \\
\hline Organic carbon & $\mathrm{OC}$ & $6.8950 \pm 3.7295$ & $58.7720 \pm 21.5960$ & $61.9970 \pm 24.9550$ & $58.3350 \pm 4.6528$ & $34.8740 \pm 2.7827$ & $62.6800 \pm 9.5480$ \\
\hline $\mathrm{OC}$ fraction 1 at $120^{\circ} \mathrm{C}$ in $\mathrm{He}$ & $\mathrm{OC} 1$ & $0.2746 \pm 0.2973$ & $24.3710 \pm 18.1950$ & $20.8160 \pm 7.6162$ & $18.2440 \pm 5.3407$ & $4.3149 \pm 0.3811$ & $10.3330 \pm 4.9033$ \\
\hline $\mathrm{OC}$ fraction 2 at $250^{\circ} \mathrm{C}$ in $\mathrm{He}$ & $\mathrm{OC} 2$ & $0.8838 \pm 0.6051$ & $12.4740 \pm 4.9880$ & $12.7670 \pm 6.2938$ & $10.2400 \pm 1.2550$ & $3.0070 \pm 0.3695$ & $10.4480 \pm 2.4690$ \\
\hline $\mathrm{OC}$ fraction 3 at $450^{\circ} \mathrm{C}$ in $\mathrm{He}$ & $\mathrm{OC} 3$ & $2.6704 \pm 1.3216$ & $13.3020 \pm 6.0825$ & $18.8010 \pm 7.2890$ & $21.2100 \pm 3.4685$ & $7.5739 \pm 0.6666$ & $26.7180 \pm 12.7580$ \\
\hline $\mathrm{OC}$ fraction 4 at $550^{\circ} \mathrm{C}$ in $\mathrm{He}$ & $\mathrm{OC} 4$ & $1.9571 \pm 0.8353$ & $7.3284 \pm 2.8507$ & $9.5810 \pm 5.4608$ & $8.6300 \pm 1.2041$ & $4.4939 \pm 0.6265$ & $8.7359 \pm 1.2399$ \\
\hline Pyrolized OC & OP & $1.1091 \pm 0.6952$ & $1.2972 \pm 2.5596$ & $0.0318 \pm 0.1382$ & $0.0117 \pm 0.0399$ & $15.4830 \pm 5.4853$ & $5.7697 \pm 2.9909$ \\
\hline Elemental carbon & EC & $0.9946 \pm 0.9520$ & $28.5650 \pm 13.8100$ & $78.3140 \pm 16.5500$ & $5.1909 \pm 0.7901$ & $27.2360 \pm 2.2356$ & $11.8760 \pm 1.4911$ \\
\hline $\mathrm{EC}$ fraction 1 at $550^{\circ} \mathrm{C}$ in $98 \% \mathrm{He} / 2 \% \mathrm{O}_{2}$ & $\mathrm{EC} 1$ & $1.0781 \pm 0.7091$ & $13.8680 \pm 6.1435$ & $26.0500 \pm 5.9936$ & $4.8393 \pm 0.9385$ & $41.2150 \pm 3.0776$ & $13.0800 \pm 3.1538$ \\
\hline $\mathrm{EC}$ fraction 2 at $700^{\circ} \mathrm{C}$ in $98 \% \mathrm{He} / 2 \% \mathrm{O}_{2}$ & $\mathrm{EC} 2$ & $1.0257 \pm 0.9381$ & $15.5220 \pm 12.9970$ & $51.9030 \pm 12.6890$ & $0.3017 \pm 0.0576$ & $1.3362 \pm 0.1858$ & $3.9735 \pm 2.6549$ \\
\hline $\mathrm{EC}$ fraction 3 at $800^{\circ} \mathrm{C}$ in $98 \% \mathrm{He} / 2 \% \mathrm{O}_{2}$ & EC3 & $0.0000 \pm 0.0823$ & $0.4739 \pm 0.3534$ & $0.3886 \pm 0.3840$ & $0.0606 \pm 0.0342$ & $0.1676 \pm 0.0525$ & $0.5915 \pm 0.5020$ \\
\hline Total carbon & $\mathrm{TC}$ & $7.8897 \pm 4.6815$ & $87.3370 \pm 25.6330$ & $140.3100 \pm 29.9440$ & $63.5260 \pm 5.0335$ & $62.1100 \pm 4.9180$ & $74.4750 \pm 10.7590$ \\
\hline Aluminum & $\mathrm{Al}$ & $10.0008 \pm 3.0147$ & $0.1073 \pm 0.0736$ & $0.1717 \pm 0.1715$ & $0.0944 \pm 0.0112$ & $0.2013 \pm 0.0176$ & $0.0508 \pm 0.0102$ \\
\hline Silicon & $\mathrm{Si}$ & $28.1663 \pm 8.9603$ & $4.7878 \pm 4.1119$ & $1.2029 \pm 0.3647$ & $0.2912 \pm 0.0230$ & $1.0151 \pm 0.0724$ & $0.5602 \pm 0.4483$ \\
\hline Phosphorus & $\mathrm{P}$ & $0.3877 \pm 0.3543$ & $0.3479 \pm 0.5129$ & $0.1782 \pm 0.0555$ & $0.0000 \pm 0.0073$ & $0.0000 \pm 0.0057$ & $0.0000 \pm 0.0061$ \\
\hline Sulfur & $\mathrm{S}$ & $0.3516 \pm 0.2100$ & $2.6670 \pm 2.4785$ & $1.4845 \pm 1.1969$ & $0.4240 \pm 0.0331$ & $0.2352 \pm 0.0169$ & $0.2427 \pm 0.0239$ \\
\hline Chlorine & $\mathrm{Cl}$ & $0.1006 \pm 0.1422$ & $0.2491 \pm 0.2978$ & $0.0768 \pm 0.0424$ & $1.3544 \pm 1.5612$ & $0.1160 \pm 0.0090$ & $1.6225 \pm 1.1894$ \\
\hline Potassium & $\mathrm{K}$ & $2.8206 \pm 0.5488$ & $0.0579 \pm 0.0474$ & $0.1096 \pm 0.0910$ & $2.9511 \pm 0.6782$ & $1.0675 \pm 0.0758$ & $0.5008 \pm 0.2895$ \\
\hline Calcium & $\mathrm{Ca}$ & $3.4850 \pm 1.1771$ & $0.7865 \pm 1.4028$ & $0.7045 \pm 0.2820$ & $0.1873 \pm 0.0225$ & $0.5216 \pm 0.0376$ & $0.1621 \pm 0.0436$ \\
\hline Titantium & $\mathrm{Ti}$ & $0.4553 \pm 0.1348$ & $0.0030 \pm 0.0569$ & $0.0153 \pm 0.0914$ & $0.0129 \pm 0.0197$ & $0.0880 \pm 0.0096$ & $0.0108 \pm 0.0287$ \\
\hline Manganese & $\mathrm{Mn}$ & $0.0759 \pm 0.0054$ & $0.0042 \pm 0.0042$ & $0.0013 \pm 0.0066$ & $0.0067 \pm 0.0007$ & $0.0129 \pm 0.0011$ & $0.0550 \pm 0.0049$ \\
\hline Iron & $\mathrm{Fe}$ & $5.2254 \pm 1.0428$ & $0.4226 \pm 0.3424$ & $0.6570 \pm 0.4100$ & $0.1402 \pm 0.0114$ & $0.5172 \pm 0.0367$ & $0.5990 \pm 0.5467$ \\
\hline Copper & $\mathrm{Cu}$ & $0.0168 \pm 0.0119$ & $0.0519 \pm 0.0537$ & $0.0157 \pm 0.0066$ & $0.0067 \pm 0.0006$ & $0.0392 \pm 0.0028$ & $0.0617 \pm 0.0067$ \\
\hline Zinc & $\mathrm{Zn}$ & $0.0965 \pm 0.0467$ & $0.4335 \pm 0.4056$ & $0.3771 \pm 0.0872$ & $0.1368 \pm 0.0135$ & $0.0925 \pm 0.0066$ & $0.0507 \pm 0.0049$ \\
\hline Arsenic & As & $0.0016 \pm 0.0027$ & $0.0001 \pm 0.0052$ & $0.0004 \pm 0.0077$ & $0.0007 \pm 0.0017$ & $0.0006 \pm 0.0016$ & $0.0019 \pm 0.0019$ \\
\hline Selenium & $\mathrm{Se}$ & $0.0002 \pm 0.0010$ & $0.0002 \pm 0.0027$ & $0.0022 \pm 0.0041$ & $0.0001 \pm 0.0007$ & $0.0000 \pm 0.0007$ & $0.0001 \pm 0.0009$ \\
\hline Bromine & $\mathrm{Br}$ & $0.0016 \pm 0.0012$ & $0.0375 \pm 0.0384$ & $0.0451 \pm 0.0711$ & $0.0045 \pm 0.0004$ & $0.0014 \pm 0.0003$ & $0.0166 \pm 0.0016$ \\
\hline Rubidium & $\mathrm{Rb}$ & $0.0139 \pm 0.0046$ & $0.0005 \pm 0.0022$ & $0.0007 \pm 0.0038$ & $0.0046 \pm 0.0005$ & $0.0019 \pm 0.0003$ & $0.0007 \pm 0.0011$ \\
\hline Strontium & $\mathrm{Sr}$ & $0.0305 \pm 0.0016$ & $0.0009 \pm 0.0023$ & $0.0029 \pm 0.0039$ & $0.0025 \pm 0.0004$ & $0.0060 \pm 0.0006$ & $0.0011 \pm 0.0011$ \\
\hline Lead & $\mathrm{Pb}$ & $0.0109 \pm 0.0074$ & $0.0257 \pm 0.0241$ & $0.0086 \pm 0.0119$ & $0.0039 \pm 0.0009$ & $0.0030 \pm 0.0008$ & $0.0082 \pm 0.0025$ \\
\hline Retene & RETENE & $0.0000 \pm 0.0000$ & $0.0042 \pm 0.0132$ & $0.0002 \pm 0.0009$ & $0.0272 \pm 0.0039$ & $0.0140 \pm 0.0012$ & $0.0059 \pm 0.0014$ \\
\hline Indeno[123-cd]pyrene & INCDPY & $0.0000 \pm 0.0000$ & $0.0340 \pm 0.0278$ & $0.0000 \pm 0.0014$ & $0.0028 \pm 0.0004$ & $0.0033 \pm 0.0005$ & $0.0053 \pm 0.0027$ \\
\hline Benzo(ghi)perylene & BGHIPE & $0.0000 \pm 0.0000$ & $0.0941 \pm 0.0827$ & $0.0000 \pm 0.0017$ & $0.0029 \pm 0.0008$ & $0.0028 \pm 0.0008$ & $0.0018 \pm 0.0035$ \\
\hline Coronene & CORONE & $0.0000 \pm 0.0000$ & $0.0836 \pm 0.0920$ & $0.0000 \pm 0.0005$ & $0.0011 \pm 0.0003$ & $0.0008 \pm 0.0003$ & $0.0001 \pm 0.0010$ \\
\hline $20 \mathrm{~S}-13 \beta(\mathrm{H}), 17 \alpha(\mathrm{H})$-diacholestane & STER35 & $0.0000 \pm 0.0000$ & $0.0068 \pm 0.0060$ & $0.0060 \pm 0.0036$ & $0.0016 \pm 0.0005$ & $0.0038 \pm 0.0009$ & $0.0000 \pm 0.0010$ \\
\hline $\mathrm{C}_{29} 20 \mathrm{~S}-13 \beta(\mathrm{H}), 17 \alpha(\mathrm{H})$-diasterane & STER45 & $0.0000 \pm 0.0000$ & $0.0182 \pm 0.0162$ & $0.0040 \pm 0.0036$ & $0.0001 \pm 0.0001$ & $0.0000 \pm 0.0001$ & $0.0000 \pm 0.0011$ \\
\hline $\mathrm{C}_{29} 20 \mathrm{~S}-13 \alpha(\mathrm{H}), 17 \beta(\mathrm{H})$-diasterane & STER48 & $0.0000 \pm 0.0000$ & $0.0031 \pm 0.0037$ & $0.0000 \pm 0.0009$ & $0.0000 \pm 0.0001$ & $0.0000 \pm 0.0001$ & $0.0000 \pm 0.0010$ \\
\hline $\mathrm{C}_{28} 20 \mathrm{R}-5 \alpha(\mathrm{H}), 14 \alpha(\mathrm{H}), 17 \alpha(\mathrm{H})$-ergostane & STER49 & $0.0000 \pm 0.0000$ & $0.0431 \pm 0.0978$ & $0.0011 \pm 0.0027$ & $0.0000 \pm 0.0001$ & $0.0000 \pm 0.0001$ & $0.0000 \pm 0.0010$ \\
\hline $17 \alpha(\mathrm{H}), 21 \beta(\mathrm{H})-29-$ Norhopane & HOP17 & $0.0000 \pm 0.0000$ & $0.0146 \pm 0.0262$ & $0.0118 \pm 0.0075$ & $0.0001 \pm 0.0002$ & $0.0000 \pm 0.0001$ & $0.0009 \pm 0.0011$ \\
\hline $17 \alpha(\mathrm{H}), 21 \beta(\mathrm{H})-29-$ Hopane & HOP19 & $0.0000 \pm 0.0000$ & $0.0446 \pm 0.0791$ & $0.0062 \pm 0.0046$ & $0.0006 \pm 0.0002$ & $0.0008 \pm 0.0003$ & $0.0026 \pm 0.0054$ \\
\hline $22 \mathrm{~S}-17 \alpha(\mathrm{H}), 21 \beta(\mathrm{H})-30,31,32$-Trishomohopane & HOP24 & $0.0000 \pm 0.0000$ & $0.0026 \pm 0.0054$ & $0.0000 \pm 0.0009$ & $0.0005 \pm 0.0003$ & $0.0005 \pm 0.0003$ & $0.0000 \pm 0.0013$ \\
\hline 22R-17 $\alpha(\mathrm{H}), 21 \beta(\mathrm{H})-30,31,32$-Trishomohopane & HOP26 & $0.0000 \pm 0.0000$ & $0.0025 \pm 0.0052$ & $0.0000 \pm 0.0009$ & $0.0001 \pm 0.0002$ & $0.0001 \pm 0.0002$ & $0.0001 \pm 0.0028$ \\
\hline Guaiacol & GUAI & $0.0000 \pm 0.0000$ & $0.0000 \pm 0.0000$ & $0.0000 \pm 0.0000$ & $0.3721 \pm 0.0309$ & $0.2409 \pm 0.0170$ & $1.6752 \pm 0.4991$ \\
\hline 4-allyl-guaiacol & ALGUAI & $0.0000 \pm 0.0000$ & $0.0000 \pm 0.0027$ & $0.0000 \pm 0.0006$ & $0.1195 \pm 0.0085$ & $0.0548 \pm 0.0055$ & $0.0067 \pm 0.0067$ \\
\hline Levoglucosan & LEVG & $0.0000 \pm 0.0000$ & $0.0000 \pm 0.0120$ & $0.0000 \pm 0.0175$ & $2.2778 \pm 0.5924$ & $0.1552 \pm 0.0172$ & $1.1505 \pm 0.4381$ \\
\hline Syringaldehyde & SYRALD & $0.0000 \pm 0.0000$ & $0.0000 \pm 0.0000$ & $0.0000 \pm 0.0000$ & $0.4631 \pm 0.0307$ & $0.0247 \pm 0.0017$ & $0.1871 \pm 0.0224$ \\
\hline Palmitoleic acid & PALOL & $0.0000 \pm 0.0000$ & $0.0082 \pm 0.0117$ & $0.0263 \pm 0.0217$ & $0.0069 \pm 0.0005$ & $0.0000 \pm 0.0002$ & $0.0261 \pm 0.0234$ \\
\hline Palmitic acid & PALAC & $0.0000 \pm 0.0000$ & $0.0000 \pm 0.0486$ & $0.0000 \pm 0.1507$ & $0.0562 \pm 0.0041$ & $0.0000 \pm 0.0323$ & $0.0000 \pm 0.1940$ \\
\hline Oleic acid & OLAC & $0.0000 \pm 0.0000$ & $0.0000 \pm 0.0152$ & $0.0000 \pm 0.0422$ & $0.0652 \pm 0.0051$ & $0.0000 \pm 0.0289$ & $0.0000 \pm 0.1385$ \\
\hline Stearic acid & STEAC & $0.0000 \pm 0.0000$ & $0.0000 \pm 0.0173$ & $0.0000 \pm 0.0358$ & $0.0174 \pm 0.0013$ & $0.0000 \pm 0.0289$ & $0.0000 \pm 0.1574$ \\
\hline Cholesterol & $\mathrm{CHOL}$ & $0.0000 \pm 0.0000$ & $0.0000 \pm 0.0011$ & $0.0000 \pm 0.0020$ & $0.0000 \pm 0.0000$ & $0.0000 \pm 0.0002$ & $0.0003 \pm 0.0012$ \\
\hline Phthalic acid & PHTHAC & $0.0000 \pm 0.0000$ & $0.1026 \pm 0.2018$ & $0.1864 \pm 0.1740$ & $0.0141 \pm 0.0010$ & $0.0000 \pm 0.0002$ & $0.0065 \pm 0.0012$ \\
\hline Norfarnesane & NORFAR & $0.0000 \pm 0.0000$ & $0.0365 \pm 0.3020$ & $0.0285 \pm 0.0236$ & $0.0020 \pm 0.0010$ & $0.0002 \pm 0.0002$ & $0.0007 \pm 0.0014$ \\
\hline Farnesane & FARNES & $0.0000 \pm 0.0000$ & $0.0344 \pm 0.5172$ & $0.0750 \pm 0.0914$ & $0.0011 \pm 0.0008$ & $0.0005 \pm 0.0005$ & $0.0000 \pm 0.0012$ \\
\hline Norpristance & NORPRI & $0.0000 \pm 0.0000$ & $0.0422 \pm 0.3857$ & $0.1178 \pm 0.0372$ & $0.0006 \pm 0.0004$ & $0.0000 \pm 0.0004$ & $0.0025 \pm 0.0034$ \\
\hline Pristane & PRIST & $0.0000 \pm 0.0000$ & $0.0032 \pm 0.2887$ & $0.0119 \pm 0.0145$ & $0.0008 \pm 0.0005$ & $0.0010 \pm 0.0004$ & $0.0352 \pm 0.0086$ \\
\hline Phytane & PHYTAN & $0.0000 \pm 0.0000$ & $0.0139 \pm 0.4463$ & $0.0974 \pm 0.0656$ & $0.0015 \pm 0.0005$ & $0.0004 \pm 0.0002$ & $0.0041 \pm 0.0021$ \\
\hline
\end{tabular}

(AGRI), dairy and feed lot (CATTLE), lake deposits (SALT), and construction (CONST). OC and EC were measured in these samples but their specific organic compounds were not measured and they are set to zero in the profile.
Examination of the ambient data for sodium $(\mathrm{Na})$ and chlorine $(\mathrm{Cl})$ (sea salt markers) showed that $\mathrm{Cl}$ was depleted with respect to $\mathrm{Na}$ in pure sea salt, even at a coastal site like Bodega Bay where the average ratio of $\mathrm{Cl} / \mathrm{Na}$ (for 
Table 1. Continued.

\begin{tabular}{|c|c|c|c|c|c|c|c|c|}
\hline \multirow[b]{2}{*}{ Chemical Species } & \multicolumn{7}{|c|}{ Source Type and Code } & \multirow[b]{2}{*}{$\begin{array}{l}\text { Ammonium Sulfate } \\
\text { AMSUL }\end{array}$} \\
\hline & $\begin{array}{l}\text { Source } \\
\text { Code }\end{array}$ & $\begin{array}{l}\text { Charbroiled Chicken } \\
\text { CHCHICK }\end{array}$ & $\begin{array}{l}\text { Propane Chicken } \\
\text { PRCHICK }\end{array}$ & $\begin{array}{l}\text { Charbroiled Hamburger } \\
\text { CHHAMB }\end{array}$ & $\begin{array}{l}\text { Meat Cooking } \\
\text { COOK }\end{array}$ & $\begin{array}{l}\text { Seasalt } \\
\text { MARINE }\end{array}$ & $\begin{array}{l}\text { Ammonium Nitrate } \\
\text { AMNIT }\end{array}$ & \\
\hline Chloride & $\mathrm{Cl}^{-}$ & $0.0449 \pm 0.0332$ & $0.5209 \pm 0.1540$ & $0.0257 \pm 0.0180$ & $0.1972 \pm 0.2805$ & $23.4880 \pm 2.6795$ & $0.0000 \pm 0.0000$ & $0.0000 \pm 0.0000$ \\
\hline Nitrate & $\mathrm{NO}_{3}^{-}$ & $0.0637 \pm 0.0291$ & $0.0855 \pm 0.0911$ & $0.0570 \pm 0.0161$ & $0.0687 \pm 0.0560$ & $41.6110 \pm 4.7469$ & $77.5000 \pm 7.7500$ & $0.0000 \pm 0.0000$ \\
\hline Sulfate & $\mathrm{SO}_{4}^{\stackrel{3}{=}}$ & $0.0950 \pm 0.0672$ & $0.2792 \pm 0.2123$ & $0.1450 \pm 0.0377$ & $0.1731 \pm 0.1304$ & $6.5468 \pm 0.7468$ & $0.0000 \pm 0.0000$ & $72.7000 \pm 7.2700$ \\
\hline Ammonium & $\mathrm{NH}_{4}^{+}$ & $0.0000 \pm 0.0289$ & $0.0000 \pm 0.0915$ & $0.0000 \pm 0.0156$ & $0.0000 \pm 0.0561$ & $0.0000 \pm 0.1000$ & $22.5500 \pm 2.2550$ & $27.3000 \pm 2.7300$ \\
\hline Water-Soluble sodium & $\mathrm{Na}^{+}$ & $0.0522 \pm 0.0069$ & $0.2508 \pm 0.0264$ & $0.0999 \pm 0.0083$ & $0.1343 \pm 0.0165$ & $26.1870 \pm 2.9874$ & $0.0000 \pm 0.0000$ & $0.0000 \pm 0.0000$ \\
\hline Water-Soluble Potassium & $\mathrm{K}^{+}$ & $0.0157 \pm 0.0038$ & $0.2647 \pm 0.0255$ & $0.0804 \pm 0.0073$ & $0.1203 \pm 0.0155$ & $0.9699 \pm 0.1106$ & $0.0000 \pm 0.0000$ & $0.0000 \pm 0.0000$ \\
\hline Organic carbon & $\mathrm{OC}$ & $68.8730 \pm 6.3751$ & $69.4010 \pm 5.8564$ & $70.0080 \pm 5.4660$ & $69.4270 \pm 5.9109$ & $0.0000 \pm 0.0000$ & $0.0000 \pm 0.0000$ & $0.0000 \pm 0.0000$ \\
\hline $\begin{array}{l}\mathrm{OC} \text { fraction } 1 \text { at } 120^{\circ} \mathrm{C} \text { in } \\
\mathrm{He}\end{array}$ & $\mathrm{OC} 1$ & $16.4470 \pm 3.7353$ & $8.2255 \pm 8.1671$ & $13.2710 \pm 2.9585$ & $12.6480 \pm 5.4591$ & $0.0000 \pm 0.0000$ & $0.0000 \pm 0.0000$ & $0.0000 \pm 0.0000$ \\
\hline $\begin{array}{l}\text { OC fraction } 2 \text { at } 250^{\circ} \mathrm{C} \text { in } \\
\mathrm{He}\end{array}$ & $\mathrm{OC} 2$ & $29.4570 \pm 6.8492$ & $20.6030 \pm 7.3992$ & $31.6790 \pm 4.3935$ & $27.2460 \pm 6.3499$ & $0.0000 \pm 0.0000$ & $0.0000 \pm 0.0000$ & $0.0000 \pm 0.0000$ \\
\hline $\begin{array}{l}\text { OC fraction } 3 \text { at } 450^{\circ} \mathrm{C} \text { in } \\
\mathrm{He}\end{array}$ & OC3 & $18.3400 \pm 2.1696$ & $32.9780 \pm 15.9640$ & $21.4670 \pm 2.5208$ & $24.2620 \pm 9.4146$ & $0.0000 \pm 0.0000$ & $0.0000 \pm 0.0000$ & $0.0000 \pm 0.0000$ \\
\hline $\begin{array}{l}\mathrm{OC} \text { fraction } 4 \text { at } 550^{\circ} \mathrm{C} \text { in } \\
\mathrm{He}\end{array}$ & $\mathrm{OC} 4$ & $3.6787 \pm 0.4984$ & $6.4667 \pm 0.9140$ & $2.8861 \pm 0.4815$ & $4.3438 \pm 1.8807$ & $0.0000 \pm 0.0000$ & $0.0000 \pm 0.0000$ & $0.0000 \pm 0.0000$ \\
\hline Pyrolized OC & OP & $0.7735 \pm 0.5627$ & $0.9295 \pm 1.0107$ & $0.6110 \pm 0.8550$ & $0.7713 \pm 0.8305$ & $0.0000 \pm 0.1000$ & $0.0000 \pm 0.0000$ & $0.0000 \pm 0.0000$ \\
\hline Elemental carbon & EC & $2.5938 \pm 0.6693$ & $11.8130 \pm 3.1734$ & $2.4850 \pm 1.8256$ & $5.6306 \pm 5.3543$ & $0.0000 \pm 0.0000$ & $0.0000 \pm 0.0000$ & $0.0000 \pm 0.0000$ \\
\hline $\begin{array}{l}\mathrm{EC} \text { fraction } 1 \text { at } 550^{\circ} \mathrm{C} \text { in } \\
98 \% \mathrm{He} / 2 \% \mathrm{O}_{2}\end{array}$ & $\mathrm{EC} 1$ & $2.8265 \pm 0.5162$ & $11.5840 \pm 4.3242$ & $2.6974 \pm 1.0513$ & $5.7025 \pm 5.0936$ & $0.0000 \pm 0.0000$ & $0.0000 \pm 0.0000$ & $0.0000 \pm 0.0000$ \\
\hline $\begin{array}{l}\mathrm{EC} \text { fraction } 2 \text { at } 700^{\circ} \mathrm{C} \text { in } \\
98 \% \mathrm{He} / 2 \% \mathrm{O}_{2}\end{array}$ & $\mathrm{EC} 2$ & $0.4571 \pm 0.0621$ & $0.9213 \pm 0.7199$ & $0.2344 \pm 0.0322$ & $0.5376 \pm 0.4176$ & $0.0000 \pm 0.0000$ & $0.0000 \pm 0.0000$ & $0.0000 \pm 0.0000$ \\
\hline $\begin{array}{l}\mathrm{EC} \text { fraction } 3 \text { at } 800^{\circ} \mathrm{C} \text { in } \\
98 \% \mathrm{He} / 2 \% \mathrm{O}_{2}\end{array}$ & $\mathrm{EC} 3$ & $0.0843 \pm 0.0495$ & $0.2385 \pm 0.1675$ & $0.1640 \pm 0.0982$ & $0.1623 \pm 0.1157$ & $0.0000 \pm 0.1000$ & $0.0000 \pm 0.0000$ & $0.0000 \pm 0.0000$ \\
\hline Total carbon & TC & $71.4450 \pm 5.7045$ & $81.1550 \pm 7.9406$ & $72.4800 \pm 5.7314$ & $75.0270 \pm 6.5433$ & $0.0000 \pm 0.0000$ & $0.0000 \pm 0.0000$ & $0.0000 \pm 0.0000$ \\
\hline Aluminum & $\mathrm{Al}$ & $0.0291 \pm 0.0032$ & $0.0082 \pm 0.0121$ & $0.0160 \pm 0.0017$ & $0.0178 \pm 0.0073$ & $0.0000 \pm 0.0000$ & $0.0000 \pm 0.0000$ & $0.0000 \pm 0.0000$ \\
\hline Silicon & $\mathrm{Si}$ & $0.1891 \pm 0.0160$ & $0.5620 \pm 0.6156$ & $0.0698 \pm 0.0055$ & $0.2736 \pm 0.3556$ & $0.0073 \pm 0.0008$ & $0.0000 \pm 0.0000$ & $0.0000 \pm 0.0000$ \\
\hline Phosphorus & $\mathrm{P}$ & $0.0002 \pm 0.0026$ & $0.0083 \pm 0.0043$ & $0.0074 \pm 0.0009$ & $0.0053 \pm 0.0029$ & $0.0001 \pm 0.0000$ & $0.0000 \pm 0.0000$ & $0.0000 \pm 0.0000$ \\
\hline Sulfur & $\mathrm{S}$ & $0.0532 \pm 0.0059$ & $0.1584 \pm 0.0173$ & $0.0797 \pm 0.0061$ & $0.0971 \pm 0.0111$ & $2.1823 \pm 0.2489$ & $0.0000 \pm 0.0000$ & $24.2700 \pm 2.4270$ \\
\hline Chlorine & $\mathrm{Cl}$ & $0.0522 \pm 0.0040$ & $0.4478 \pm 0.0326$ & $0.0636 \pm 0.0047$ & $0.1879 \pm 0.0192$ & $23.4880 \pm 2.6795$ & $0.0000 \pm 0.0000$ & $0.0000 \pm 0.0000$ \\
\hline Potassium & $\mathrm{K}$ & $0.0386 \pm 0.0032$ & $0.3212 \pm 0.0236$ & $0.0990 \pm 0.0073$ & $0.1529 \pm 0.0144$ & $0.9699 \pm 0.1106$ & $0.0000 \pm 0.0000$ & $0.0000 \pm 0.0000$ \\
\hline Calcium & $\mathrm{Ca}$ & $0.1658 \pm 0.0169$ & $0.0783 \pm 0.0336$ & $0.0987 \pm 0.0091$ & $0.1143 \pm 0.0223$ & $0.9990 \pm 0.1140$ & $0.0000 \pm 0.0000$ & $0.0000 \pm 0.0000$ \\
\hline Titantium & $\mathrm{Ti}$ & $0.0030 \pm 0.0083$ & $0.0049 \pm 0.0235$ & $0.0011 \pm 0.0045$ & $0.0030 \pm 0.0146$ & $0.0000 \pm 0.0000$ & $0.0000 \pm 0.0000$ & $0.0000 \pm 0.0000$ \\
\hline Manganese & $\mathrm{Mn}$ & $0.0098 \pm 0.0009$ & $0.0074 \pm 0.0011$ & $0.0066 \pm 0.0005$ & $0.0079 \pm 0.0009$ & $0.0000 \pm 0.0000$ & $0.0000 \pm 0.0000$ & $0.0000 \pm 0.0000$ \\
\hline Iron & $\mathrm{Fe}$ & $0.1430 \pm 0.0128$ & $0.1049 \pm 0.0090$ & $0.0558 \pm 0.0041$ & $0.1012 \pm 0.0093$ & $0.0000 \pm 0.0000$ & $0.0000 \pm 0.0000$ & $0.0000 \pm 0.0000$ \\
\hline Copper & $\mathrm{Cu}$ & $0.0089 \pm 0.0008$ & $0.0077 \pm 0.0013$ & $0.0033 \pm 0.0004$ & $0.0066 \pm 0.0009$ & $0.0000 \pm 0.0000$ & $0.0000 \pm 0.0000$ & $0.0000 \pm 0.0000$ \\
\hline Zinc & $\mathrm{Zn}$ & $0.0134 \pm 0.0010$ & $0.0089 \pm 0.0011$ & $0.0051 \pm 0.0004$ & $0.0091 \pm 0.0009$ & $0.0000 \pm 0.0000$ & $0.0000 \pm 0.0000$ & $0.0000 \pm 0.0000$ \\
\hline Arsenic & As & $0.0002 \pm 0.0094$ & $0.0002 \pm 0.0015$ & $0.0001 \pm 0.0003$ & $0.0002 \pm 0.0055$ & $0.0000 \pm 0.0000$ & $0.0000 \pm 0.0000$ & $0.0000 \pm 0.0000$ \\
\hline Selenium & $\mathrm{Se}$ & $0.0000 \pm 0.0003$ & $0.0001 \pm 0.0006$ & $0.0000 \pm 0.0001$ & $0.0000 \pm 0.0004$ & $0.0000 \pm 0.0000$ & $0.0000 \pm 0.0000$ & $0.0000 \pm 0.0000$ \\
\hline Bromine & $\mathrm{Br}$ & $0.0006 \pm 0.0007$ & $0.0018 \pm 0.0005$ & $0.0010 \pm 0.0001$ & $0.0011 \pm 0.0005$ & $0.1625 \pm 0.0185$ & $0.0000 \pm 0.0000$ & $0.0000 \pm 0.0000$ \\
\hline Rubidium & $\mathrm{Rb}$ & $0.0002 \pm 0.0003$ & $0.0005 \pm 0.0008$ & $0.0000 \pm 0.0002$ & $0.0002 \pm 0.0005$ & $0.0000 \pm 0.0000$ & $0.0000 \pm 0.0000$ & $0.0000 \pm 0.0000$ \\
\hline Strontium & $\mathrm{Sr}$ & $0.0015 \pm 0.0003$ & $0.0004 \pm 0.0010$ & $0.0006 \pm 0.0002$ & $0.0008 \pm 0.0006$ & $0.0192 \pm 0.0022$ & $0.0000 \pm 0.0000$ & $0.0000 \pm 0.0000$ \\
\hline Lead & $\mathrm{Pb}$ & $0.0434 \pm 0.0044$ & $0.0000 \pm 0.0025$ & $0.0000 \pm 0.0005$ & $0.0145 \pm 0.0029$ & $0.0000 \pm 0.0000$ & $0.0000 \pm 0.0000$ & $0.0000 \pm 0.0000$ \\
\hline Retene & RETENE & $0.0012 \pm 0.0004$ & $0.0025 \pm 0.0012$ & $0.0006 \pm 0.0002$ & $0.0014 \pm 0.0007$ & $0.0000 \pm 0.0000$ & $0.0000 \pm 0.0000$ & $0.0000 \pm 0.0000$ \\
\hline Indeno[123-cd]pyrene & INCDPY & $0.0005 \pm 0.0007$ & $0.0028 \pm 0.0022$ & $0.0003 \pm 0.0004$ & $0.0012 \pm 0.0014$ & $0.0000 \pm 0.0000$ & $0.0000 \pm 0.0000$ & $0.0000 \pm 0.0000$ \\
\hline Benzo(ghi)perylene & BGHIPE & $0.0003 \pm 0.0009$ & $0.0068 \pm 0.0034$ & $0.0008 \pm 0.0005$ & $0.0026 \pm 0.0021$ & $0.0000 \pm 0.0000$ & $0.0000 \pm 0.0000$ & $0.0000 \pm 0.0000$ \\
\hline Coronene & CORONE & $0.0000 \pm 0.0003$ & $0.0029 \pm 0.0013$ & $0.0000 \pm 0.0002$ & $0.0010 \pm 0.0008$ & $0.0000 \pm 0.0000$ & $0.0000 \pm 0.0000$ & $0.0000 \pm 0.0000$ \\
\hline $\begin{array}{l}\text { 20S-13 } \beta(\mathrm{H}), 17 \alpha(\mathrm{H})- \\
\text { diacholestane }\end{array}$ & STER35 & $0.0000 \pm 0.0003$ & $0.0000 \pm 0.0008$ & $0.0000 \pm 0.0002$ & $0.0000 \pm 0.0005$ & $0.0000 \pm 0.0000$ & $0.0000 \pm 0.0000$ & $0.0000 \pm 0.0000$ \\
\hline $\begin{array}{l}\mathrm{C}_{29} 20 \mathrm{~S}-13 \beta(\mathrm{H}), \quad 17 \alpha(\mathrm{H})- \\
\text { diasterane }\end{array}$ & STER45 & $0.0000 \pm 0.0003$ & $0.0000 \pm 0.0008$ & $0.0000 \pm 0.0002$ & $0.0000 \pm 0.0005$ & $0.0000 \pm 0.0000$ & $0.0000 \pm 0.0000$ & $0.0000 \pm 0.0000$ \\
\hline $\begin{array}{l}\mathrm{C}_{29} 20 \mathrm{~S}-13 \alpha(\mathrm{H}), \quad 17 \beta(\mathrm{H})- \\
\text { diasterane }\end{array}$ & STER48 & $0.0000 \pm 0.0003$ & $0.0000 \pm 0.0008$ & $0.0000 \pm 0.0002$ & $0.0000 \pm 0.0005$ & $0.0000 \pm 0.0000$ & $0.0000 \pm 0.0000$ & $0.0000 \pm 0.0000$ \\
\hline $\begin{array}{l}\mathrm{C}_{28} 20 \mathrm{R}-5 \alpha(\mathrm{H}) \\
14 \alpha(\mathrm{H}), 17 \alpha(\mathrm{H}) \text {-ergostane }\end{array}$ & STER49 & $0.0000 \pm 0.0003$ & $0.0000 \pm 0.0008$ & $0.0001 \pm 0.0002$ & $0.0000 \pm 0.0005$ & $0.0000 \pm 0.0000$ & $0.0000 \pm 0.0000$ & $0.0000 \pm 0.0000$ \\
\hline $\begin{array}{l}17 \alpha(\mathrm{H}), \\
\text { Norhopane }\end{array}$ & HOP17 & $0.0002 \pm 0.0003$ & $0.0007 \pm 0.0009$ & $0.0001 \pm 0.0002$ & $0.0003 \pm 0.0006$ & $0.0000 \pm 0.0000$ & $0.0000 \pm 0.0000$ & $0.0000 \pm 0.0000$ \\
\hline $\begin{array}{l}17 \alpha(\mathrm{H}), \\
\text { Hopane }\end{array}$ & HOP19 & $0.0000 \pm 0.0011$ & $0.0000 \pm 0.0023$ & $0.0003 \pm 0.0008$ & $0.0001 \pm 0.0015$ & $0.0000 \pm 0.0000$ & $0.0000 \pm 0.0000$ & $0.0000 \pm 0.0000$ \\
\hline $\begin{array}{l}22 \mathrm{~S}-17 \alpha(\mathrm{H}), 21 \beta(\mathrm{H})- \\
30,31,32 \text {-Trishomohopane }\end{array}$ & HOP24 & $0.0000 \pm 0.0004$ & $0.0000 \pm 0.0008$ & $0.0000 \pm 0.0002$ & $0.0000 \pm 0.0005$ & $0.0000 \pm 0.0000$ & $0.0000 \pm 0.0000$ & $0.0000 \pm 0.0000$ \\
\hline $\begin{array}{l}22 \mathrm{R}-17 \alpha(\mathrm{H}), 21 \beta(\mathrm{H})- \\
30,31,32 \text {-Trishomohopane }\end{array}$ & HOP26 & $0.0000 \pm 0.0004$ & $0.0000 \pm 0.0008$ & $0.0000 \pm 0.0002$ & $0.0000 \pm 0.0005$ & $0.0000 \pm 0.0000$ & $0.0000 \pm 0.0000$ & $0.0000 \pm 0.0000$ \\
\hline Guaiacol & GUAI & $0.0014 \pm 0.0013$ & $0.0015 \pm 0.0026$ & $0.0060 \pm 0.0025$ & $0.0030 \pm 0.0026$ & $0.0000 \pm 0.0000$ & $0.0000 \pm 0.0000$ & $0.0000 \pm 0.0000$ \\
\hline 4-allyl-guaiacol & ALGUAI & $0.0000 \pm 0.0003$ & $0.0000 \pm 0.0033$ & $0.0000 \pm 0.0006$ & $0.0000 \pm 0.0019$ & $0.0000 \pm 0.0000$ & $0.0000 \pm 0.0000$ & $0.0000 \pm 0.0000$ \\
\hline Levoglucosan & LEVG & $0.0135 \pm 0.0017$ & $0.0274 \pm 0.0036$ & $0.0159 \pm 0.0020$ & $0.0189 \pm 0.0026$ & $0.0000 \pm 0.0000$ & $0.0000 \pm 0.0000$ & $0.0000 \pm 0.0000$ \\
\hline Syringaldehyde & SYRALD & $0.0016 \pm 0.0004$ & $0.0046 \pm 0.0010$ & $0.0015 \pm 0.0003$ & $0.0026 \pm 0.0018$ & $0.0000 \pm 0.0000$ & $0.0000 \pm 0.0000$ & $0.0000 \pm 0.0000$ \\
\hline Palmitoleic acid & PALOL & $0.0694 \pm 0.0499$ & $0.1179 \pm 0.0795$ & $0.0410 \pm 0.0243$ & $0.0761 \pm 0.0560$ & $0.0000 \pm 0.0000$ & $0.0000 \pm 0.0000$ & $0.0000 \pm 0.0000$ \\
\hline Palmitic acid & PALAC & $0.0593 \pm 0.1499$ & $0.0001 \pm 0.2811$ & $0.0508 \pm 0.1037$ & $0.0367 \pm 0.1934$ & $0.0000 \pm 0.0000$ & $0.0000 \pm 0.0000$ & $0.0000 \pm 0.0000$ \\
\hline Oleic acid & OLAC & $0.1813 \pm 0.1632$ & $0.2741 \pm 0.3188$ & $0.1965 \pm 0.1239$ & $0.2173 \pm 0.2188$ & $0.0000 \pm 0.0000$ & $0.0000 \pm 0.0000$ & $0.0000 \pm 0.0000$ \\
\hline Stearic acid & STEAC & $0.0000 \pm 0.0565$ & $0.0000 \pm 0.2642$ & $0.0027 \pm 0.0693$ & $0.0009 \pm 0.1610$ & $0.0000 \pm 0.0000$ & $0.0000 \pm 0.0000$ & $0.0000 \pm 0.0000$ \\
\hline Cholesterol & CHOL & $0.0220 \pm 0.0058$ & $0.0373 \pm 0.0088$ & $0.0283 \pm 0.0053$ & $0.0292 \pm 0.0068$ & $0.0000 \pm 0.0000$ & $0.0000 \pm 0.0000$ & $0.0000 \pm 0.0000$ \\
\hline Phthalic acid & PHTHAC & $0.0009 \pm 0.0003$ & $0.0078 \pm 0.0013$ & $0.0000 \pm 0.0002$ & $0.0029 \pm 0.0008$ & $0.0000 \pm 0.0000$ & $0.0000 \pm 0.0000$ & $0.0000 \pm 0.0000$ \\
\hline Norfarnesane & NORFAR & $0.0000 \pm 0.0003$ & $0.0000 \pm 0.0008$ & $0.0000 \pm 0.0002$ & $0.0000 \pm 0.0005$ & $0.0000 \pm 0.0000$ & $0.0000 \pm 0.0000$ & $0.0000 \pm 0.0000$ \\
\hline Farnesane & FARNES & $0.0000 \pm 0.0003$ & $0.0000 \pm 0.0008$ & $0.0003 \pm 0.0004$ & $0.0001 \pm 0.0005$ & $0.0000 \pm 0.0000$ & $0.0000 \pm 0.0000$ & $0.0000 \pm 0.0000$ \\
\hline Norpristance & NORPRI & $0.0059 \pm 0.0018$ & $0.0010 \pm 0.0026$ & $0.0112 \pm 0.0022$ & $0.0060 \pm 0.0022$ & $0.0000 \pm 0.0000$ & $0.0000 \pm 0.0000$ & $0.0000 \pm 0.0000$ \\
\hline Pristane & PRIST & $0.0112 \pm 0.0032$ & $0.0135 \pm 0.0043$ & $0.0238 \pm 0.0048$ & $0.0162 \pm 0.0042$ & $0.0000 \pm 0.0000$ & $0.0000 \pm 0.0000$ & $0.0000 \pm 0.0000$ \\
\hline Phytane & PHYTAN & $0.0018 \pm 0.0006$ & $0.0001 \pm 0.0013$ & $0.0012 \pm 0.0004$ & $0.0010 \pm 0.0009$ & $0.0000 \pm 0.0000$ & $0.0000 \pm 0.0000$ & $0.0000 \pm 0.0000$ \\
\hline
\end{tabular}


Table 2. Average absolute error (AAE \%) between the CMB estimated and true source contribution estimates from simulated data.

\begin{tabular}{|c|c|c|c|c|c|c|c|c|c|c|c|c|c|c|}
\hline \multirow[b]{2}{*}{ Case } & \multirow[b]{2}{*}{ Organics } & \multirow[b]{2}{*}{$\begin{array}{l}\text { Ambient } \\
\text { Uncert. }\end{array}$} & \multirow[b]{2}{*}{$\begin{array}{l}\text { Source } \\
\text { Uncert. }\end{array}$} & \multicolumn{11}{|c|}{ AAE $(\%)$ by Source code ${ }^{\mathrm{a}}$} \\
\hline & & & & PVRD & GAS & DIES & MOBILE $^{b}$ & BURN-H & BURN-S & BURN $^{c}$ & COOK & MARINE & AMSUL & AMNIT \\
\hline 1 & $\mathrm{~N}$ & $10 \%$ & $30 \%$ & 26 & 84 & 19 & 15 & 13 & 34 & 10 & 45 & 107 & 24 & 18 \\
\hline 2 & $\mathrm{Y}$ & $10 \%$ & $30 \%$ & 22 & 10 & 8 & 7 & 10 & 29 & 8 & 14 & 103 & 23 & 18 \\
\hline 3 & $\mathrm{~N}$ & Actual & Actual & 72 & 178 & 29 & 33 & 17 & 108 & 20 & 70 & 268 & 18 & 8 \\
\hline 4 & $\mathrm{Y}$ & Actual & Actual & 67 & 52 & 21 & 19 & 13 & 98 & 16 & 20 & 272 & 16 & 8 \\
\hline 5 & $\mathrm{Y}$ & Actual & Actual & 76 & 50 & 21 & 16 & 20 & - & 20 & 21 & 282 & 16 & 8 \\
\hline 6 & $\mathrm{Y}$ & Actual & Actual & 58 & 50 & 19 & 17 & 8 & - & 8 & 20 & 210 & 16 & 7 \\
\hline
\end{tabular}

${ }^{a}$ See Table 1 for source codes

$\mathrm{b}$ MOBILE $=$ GAS+DIES

c BURN=BURN-H+BURN-S

Case 1: Data generated with BURN-H (hardwood) and BURN-S (softwood), no organics in CMB.

Case 2: Data generated with BURN-H (hardwood) and BURN-S (softwood), organics in CMB.

Case 3: Data generated with BURN-H (hardwood) and BURN-S (softwood), no organics in CMB.

Case 4: Data generated with BURN-H (hardwood) and BURN-S (softwood), organics in CMB.

Case 5: Data generated with BURN-H (hardwood) and BURN-S (softwood), organics in CMB, no BURN-S in CMB.

Case 6: Data generated with BURN-H (hardwood) only, organics in CMB.

concentrations greater than their uncertainties) was $1.1 \mathrm{com}$ pared with a pure sea salt ratio of 1.8. This depletion results from reactions of sea salt particles with strong acids like $\mathrm{HNO}_{3}$, where $\mathrm{NO}_{3}^{-}$substitutes for $\mathrm{Cl}$ (Mamane and Gottlieb, 1992). To account for this, a "reacted" sea salt profile (MARINE) was used in which half of the $\mathrm{Cl}$ was replaced by $\mathrm{NO}_{3}^{-}$ on a molar basis (Chow et al., 1996). Secondary $\mathrm{NO}_{3}^{-}$and $\mathrm{SO}_{4}^{=}$were represented by pure ammonium nitrate (AMNIT; $\left.\mathrm{NH}_{4} \mathrm{NO}_{3}\right)$ and ammonium sulfate [AMSUL; $\left(\mathrm{NH}_{4}\right)_{2} \mathrm{SO}_{4}$ ] profiles, respectively.

\section{Results and discussion}

\subsection{CMB feasibility analysis}

Simulated data were generated with methods described by Javitz et al. (1988), Lowenthal et al. (1992), and Chow et al. (2004b). Average true source contributions from PVRD, GAS, DIES, BURN-H, BURN-S, COOK, MARINE, AMSUL, and AMNIT of 1, 3, 10, 30, 10, 10, 0.1, 5, and $30 \mu \mathrm{g} / \mathrm{m}^{3}$, respectively, were based on previous SJV source apportionments studies. True $S_{j t}$ were created by randomly perturbing the average values (above) with a coefficient of variation $(\mathrm{CV})$ of $50 \%$, assuming a lognormal distribution. Synthetic concentrations were calculated for each "sample" using Eq. (1). Random lognormal variation for the source profiles (F) and measurement uncertainty was introduced to the derived concentrations (C) in two ways: 1) assuming measurement uncertainty and source profile variations of 10 and $30 \%$, respectively; and 2) using the root-mean squared uncertainties of ambient concentrations and the actual standard deviations of the composite source profiles. The latter approach may be more realistic because some species are measured more precisely than others. Cholesterol levels were below lower quantifiable limits (LQLs) in many of the samples owing to the short sample durations and periods of the day when cooking contributions were not expected. Cholesterol has also been reported to react with ozone under ambient conditions (Dreyfus et al., 2005). However, cholesterol was well-determined in the meat cooking emissions samples. To allow this compound to act as a useful marker for cooking in the simulations, its uncertainty in the ambient measurements was assumed to be $10 \%$.

The CMB model was applied to the two data sets, each with 100 simulated samples using the average source profiles with weighting based on the uncertainties described above. The variance of the $S_{j t}$ is the precision attainable for a particular source mix for a model with specified random errors. This precision is expressed as the average absolute error (AAE \%), which is the average $(\mathrm{N}=100)$ of the absolute percent differences between the estimated and true $S_{j t}$. Results are summarized in Table 2.

Case 1 represents fixed uncertainty without organics. The $S_{\text {MARINE }}$ AAE was large $(107 \%)$ because the true average $S_{\text {MARINE }}$ was only $0.1 \mu \mathrm{g} / \mathrm{m}^{3}$. The AAEs for $S_{\text {DIES }}$ and $S_{\mathrm{BURN}-\mathrm{H}}$ were less than $20 \%$ while the AAEs for $S_{\mathrm{GAS}}, S_{\mathrm{BURN}-\mathrm{S}}$, and $S_{\mathrm{COOK}}$ were 84,34 and $45 \%$, respectively. When organics were included (Case 2), the AAEs were much lower for $S_{\mathrm{GAS}}, S_{\mathrm{DIES}}$, and $S_{\mathrm{COOK}}$, but they did not change as much for $S_{\mathrm{BURN}-\mathrm{H}}$ and $S_{\mathrm{BURN}-S}$. Including organic compounds reduced collinearity (similarity) among profiles for the vehicle exhaust and cooking sources. Except for $S_{\mathrm{BURN}-\mathrm{H}}, S_{\mathrm{AMSUL}}$, and $S_{\mathrm{AMNIT}}$, the AAEs for Case 3 (no organics) were considerably larger than for Case 1: 72, 178, 29, 108, 70, and 268\% for contributions from PVRD, GAS, DIES, BURN-S, COOK, and 
MARINE, respectively. Including organics (Case 4) reduced the $S_{\mathrm{GAS}}, S_{\mathrm{DIES}}, S_{\mathrm{BURN}-\mathrm{H}}$, and $S_{\mathrm{COOK}}$ AAEs to $52,21,13$, and $20 \%$, respectively. While the $S_{\mathrm{BURN}-\mathrm{H}} \mathrm{AAE}$ improved somewhat (from $17 \%$ to $13 \%$ ) when organics were included, the $S_{\text {BURN-S }}$ AAE remained high (98\%).

These results verify that organic markers can help distinguish contributions from gasoline exhaust, diesel exhaust, and cooking by increasing the differences between their source profiles. However, organics were not needed to estimate the wood burning contribution. Organics did not appear to separate hardwood and softwood contributions, even though there are noticeable differences between their source profiles. For example, the $\mathrm{OC}, \mathrm{EC}, \mathrm{K}^{+}$, levoglucosan, 4-allyl-guaiacol, and syringaldehyde compositions of hardwood smoke were 58, 5.2, 2.9, 2.3, 0.12, and $0.46 \%$, respectively, compared with $35,27,0.81,0.16,0.055$, and $0.025 \%$, respectively, for softwood smoke. Case 5 demonstrates the collinearity between the hardwood and softwood profiles by removing BURN-S from the CMB fit. Even though softwood combustion emissions contributed to the simulated concentrations, the hardwood profile (BURN-H) was sufficient to estimate the total burning contribution to within $20 \%$. When all of the actual burning contribution came from hardwood combustion, the $S_{\mathrm{BURN}-\mathrm{H}}$ AAE was only $8 \%$.

The CMB8 model output contains the diagnostic MPIN (modified pseudo-inverse normalized) matrix (Kim and Henry, 1999). The MPIN identifies the influence of the fitting species on the source contribution estimates. An MPIN value of one indicates the highest influence. Average concentrations from Case 4, Table 2 were subjected to CMB analysis and the MPIN was calculated. The influential species in the source profiles were as expected: $\mathrm{Al}$ and $\mathrm{Si}$ for PVRD (paved road); benzo(ghi)perylene, coronene, and indeno[123-cd]pyrene for gasoline vehicles; the EC2 thermal fraction for diesel vehicles; $\mathrm{K}^{+}$, levoglucosan, and syringaldehyde for hardwood combustion; EC for softwood combustion; and cholesterol for cooking.

These tests with simulated data demonstrate the feasibility of identifying and quantifying gasoline- and diesel-exhaust contributions with reasonable precision using organic markers. This is also the case for cooking contributions. Organics were not necessary to estimate the RWC contribution and it was not feasible to distinguish hardwood and softwood contributions from the source profiles used in this study, even when organics were included in the CMB model.

\subsection{Initial source contribution estimates}

Following the CMB applications and validation protocol (Watson et al., 1998b), the stability of the $S_{j t}$ to different selections of source profiles and fitting species was evaluated for the average concentrations for the 00:00-05:00 PST sampling period. Ambient concentrations during this interval, including those of levoglucosan and cholesterol, markers for RWC and cooking, respectively, were relatively high
Table 3. Fitting species ${ }^{\mathrm{a}}$ used in CMB modeling for Fresno winter intensive samples.

\begin{tabular}{ll}
\hline Traditional species & Organic species \\
\hline $\mathrm{NO}_{3}^{-}$ & Indeno[123-cd]pyrene (INCDPY) \\
$\mathrm{SO}_{4}^{\overline{-}}$ & Benzo(ghi)perylene (BGHIPE) \\
$\mathrm{NH}_{4}^{+}$ & Coronene (CORONE) \\
$\mathrm{Na}^{+}$ & $17 \mathrm{a}(\mathrm{H}), 213(\mathrm{H})-29-H o p a n e(H O P 17)$ \\
$\mathrm{K}^{+}$(soluble K) & Levoglucosan (LEVG) \\
$\mathrm{OC}^{+}$ & Syringaldehyde (SYRALD) \\
$\mathrm{OC} 4$ & Palmitoleic acid (PALOL) \\
$\mathrm{OC}$ & Oleic acid (OLAC) \\
$\mathrm{EC} 2$ & Cholesterol (CHOL) \\
$\mathrm{EC} 3$ & Norfarnesane (NORFAR) \\
$\mathrm{EC}$ & Farnesane (FARNES) \\
$\mathrm{Al}$ & Norpristane (NORPRI) \\
$\mathrm{Si}$ & Pristane (PRIST) \\
$\mathrm{Cl}$ & Phytane (PHYTAN) \\
$\mathrm{K}($ total K) & \\
$\mathrm{Fe}$ & \\
$\mathrm{Se}$ & \\
$\mathrm{Br}$ & \\
$\mathrm{Pb}$ & \\
\hline
\end{tabular}

a See Table 1 for chemical species.

and it is expected that this period is not dominated by a single source contribution. Chemical species whose concentrations were less than their uncertainties in most samples (more than 40 out of 51 total sampling periods in Fresno) were not included in the CMB model. While cholesterol did not fit this criterion, it was included because of its potential value as a cooking marker. Initial model runs indicated that other species were not adequately accounted for in the CMB. Calcium $(\mathrm{Ca})$, whose concentrations were greater than twice their uncertainties in only 15 out of 51 samples, was overestimated by a factor of 5. Copper $(\mathrm{Cu})$ and zinc $(\mathrm{Zn})$ could not be explained by the available source profiles, including municipal incineration and brake wear. These species may be enriched by exhaust from the sampling equipment (Hoffman and Duce, 1971; King and Toma, 1975; Patterson, 1980). Guaiacol and 4-allyl-guaiacol, potential RWC markers, were underestimated by factors of 2 to 10 . This could be attributed to differences between the profile fuels and burning conditions and those used in Fresno. Thermal carbon fractions were included except for OP (pyrolized OC), OC1 and OC2, which are believed to contain much of the adsorbed organic vapors on quartz filters, and EC1, which may contain some pyrolysis products. Table 3 shows the 19 traditional and 14 organic species included in subsequent CMB analyses.

Case 1 in Table 4 gives the CMB solution for the "best fit", which included organic species and both hardwood and softwood RWC source profiles. In a statistical sense, it is not clear that the BURN-S contribution was resolved because its 
Table 4. Source contribution estimates from the CMB trial runs for average Fresno winter intensive samples during the early morning (00:00-05:00 PST) period, with and without organics for various source mixes.

\begin{tabular}{|c|c|c|c|c|c|c|c|c|c|c|c|c|}
\hline \multirow[b]{2}{*}{ Case } & \multicolumn{12}{|c|}{ Source contributions $\left(\mu \mathrm{g} / \mathrm{m}^{3}\right)$} \\
\hline & PVRD & GAS & DIES & BURN-H & BURN-S & COOK & MARINE & AMSUL & AMNIT & PCMASS & R SQR & CHI SQR \\
\hline $1^{\mathrm{a}}$ & 0 & $1.9 \pm 1.3$ & $6.6 \pm 2.2$ & $16 \pm 3$ & $5.8 \pm 6.2$ & $20 \pm 5$ & 0 & $1.1 \pm 0.4$ & $18 \pm 2$ & 92 & 0.96 & 0.6 \\
\hline $2^{\mathrm{b}}$ & 0 & 0 & $7.1 \pm 2.3$ & $15 \pm 3$ & $7.0 \pm 6.4$ & $23 \pm 6$ & 0 & $1.3 \pm 0.3$ & $18 \pm 2$ & 94 & 0.98 & 0.7 \\
\hline $3^{\mathrm{a}}$ & 0 & $2.2 \pm 1.4$ & $7.6 \pm 2.2$ & $18 \pm 2$ & - & $21 \pm 6$ & 0 & $1.1 \pm 0.4$ & $18 \pm 2$ & 89 & 0.96 & 0.6 \\
\hline $4^{\mathrm{b}}$ & $0.04 \pm 0.3$ & 0 & $8.5 \pm 2.2$ & $17 \pm 2$ & - & $25 \pm 6$ & 0 & $1.3 \pm 0.4$ & $18 \pm 2$ & 91 & 0.97 & 0.7 \\
\hline $5^{a}$ & 0 & $1.0 \pm 0.9$ & $3.0 \pm 1.6$ & - & $37 \pm 3$ & $23 \pm 5$ & $0.49 \pm 0.12$ & $1.3 \pm 0.3$ & $18 \pm 2$ & 110 & 0.88 & 3.0 \\
\hline $6^{\mathrm{b}}$ & 0 & 0 & $3.2 \pm 1.6$ & - & $36 \pm 3$ & $24 \pm 6$ & $0.49 \pm 0.12$ & $1.4 \pm 0.3$ & $18 \pm 2$ & 109 & 0.91 & 4.1 \\
\hline $7^{\mathrm{a}}$ & 0 & $2.4 \pm 1.4$ & $8.2 \pm 2.4$ & $19 \pm 3$ & $10 \pm 6$ & - & 0 & $1.0 \pm 0.4$ & $18 \pm 2$ & 77 & 0.92 & 1.2 \\
\hline $8^{\mathrm{b}}$ & 0 & $30 \pm 7$ & 0 & $18 \pm 2$ & 0 & - & $0.05 \pm 0.20$ & 0 & $17 \pm 2$ & 85 & 0.97 & 0.4 \\
\hline
\end{tabular}

a With organics.

b Without organics.

value was lower than its uncertainty. On the other hand, including this source accounted for a larger percentage of the measured mass. The best estimate of the RWC contribution may be the sum of $S_{\mathrm{BURN}-\mathrm{H}}$ and $S_{\mathrm{BURN}-\mathrm{S}}\left(22 \pm 7 \mu \mathrm{g} / \mathrm{m}^{3}\right)$. Similarly, while GAS and DIES contributions were resolved, the uncertainty of $S_{\mathrm{GAS}}\left(1.9 \pm 1.3 \mu \mathrm{g} / \mathrm{m}^{3}\right)$ was large $(68 \%)$. The cooking contribution was large $\left(20 \pm 5 \mu \mathrm{g} / \mathrm{m}^{3}\right)$ as was the secondary $\mathrm{NH}_{4} \mathrm{NO}_{3}$ contribution $\left(18 \pm 2 \mu \mathrm{g} / \mathrm{m}^{3}\right)$. Zero values for $S_{\mathrm{PVRD}}$ and $S_{\mathrm{MARINE}}$ indicate that their contributions became negative in the iterative solution and that their respective source profiles were dropped from the model. Most of the measured mass was accounted for (PCMASS=92) and the included sources explained the ambient chemical concentrations well (R SQR=0.96, CHI SQR=0.6).

The distinguishing chemical markers for the sources in Case 1 were examined with the MPIN matrix, a feature of the CMB8 model, shown in Table 5. According to the MPIN, the most important markers for cooking were OC, OC3, and palmitoleic acid. Cholesterol exhibited a relatively low value because its average ambient concentration was smaller than its uncertainty. The MPIN indicated that the most important GAS markers were coronene and benzo(ghi)perylene, as expected. The EC2 fraction was the most important DIES marker. The principal hardwood (BURN-H) markers were $\mathrm{K}^{+}$and syringaldehyde. Levoglucosan was also an important marker with a value of 0.5 . The MPIN shows that the most influential marker for softwood (BURN-S) was Fe, but this should not be the case.

Case 2 (Table 4) was the same as Case 1 except that organic species were excluded from the fit. Except for a $S_{\mathrm{GAS}}$ of zero, the solution was very similar to Case 1 (with organics) although $S_{\text {COOK was }} 3 \mu \mathrm{g} / \mathrm{m}^{3}$ higher. Cases 3 and 4 were analogous to Cases 1 and 2, respectively, except that BURN$\mathrm{S}$ was removed from the model. In Case 3, with organics, removing BURN-S increased the $S_{\mathrm{GAS}}$ and $S_{\mathrm{DIES}}$ slightly and increased $S_{\mathrm{BURN}-\mathrm{H}}$ and $S_{\mathrm{COOK}}$ by 2 and $1 \mu \mathrm{g} / \mathrm{m}^{3}$, respectively. In Case 4 (without organics), all of the vehicle exhaust contribution was assigned to DIES, as in Case 2, and $S_{\text {COOK }}$ increased from $23 \pm 6$ (Case 2) to $25 \pm 6 \mu \mathrm{g} / \mathrm{m}^{3}$. Removing BURN-S in Cases 3 and 4 reduced PCMASS by $3 \%$ and most of this decrease came from the burning source contribution.

Case 5 (with organics) and Case 6 (without organics) were analogous to Cases 3 and 4, respectively, except that BURN$\mathrm{S}$ was included and BURN-H was excluded from the model. This caused a large increase in the burning contribution, to $37 \pm 3$ and $36 \pm 3 \mu \mathrm{g} / \mathrm{m}^{3}$, with and without organics, respectively, and an overestimation of measured mass by 10 and $9 \%$, respectively. Both $S_{\mathrm{GAS}}$ and $S_{\mathrm{DIES}}$ were reduced by about a factor of 2 and $S_{\mathrm{COOK}}$ increased by $3 \mu \mathrm{g} / \mathrm{m}^{3}$ compared with Case 1 . The R SQR decreased and CHI SQR increased dramatically compared with previous cases, indicating that BURN-S did not explain the traditional or organic species concentrations as well as BURN-H.

Finally, the cooking profile was removed while BURN$\mathrm{H}$ and BURN-S were retained. In Case 7 (with organics), the solution was similar to that of Case 1 although $S_{\mathrm{GAS}}$ and $S_{\text {DIES }}$ increased somewhat while the total burning contribution increased from $22 \pm 7$ to $29 \pm 7 \mu \mathrm{g} / \mathrm{m}^{3}$. The solution changed dramatically without organics (Case 8). All of $S_{\mathrm{BURN}-\mathrm{S}}$ and $S_{\mathrm{COOK}}$ were assigned to $S_{\mathrm{GAS}}\left(30 \pm 7 \mu \mathrm{g} / \mathrm{m}^{3}\right)$. Both DIES and BURN-S were eliminated from the fit. Note that while mass was underestimated by $15 \%$, this model fit the non-organic concentrations well (R SQR $=0.97$, CHI $\mathrm{SQR}=0.4$ ). However, the previous results suggest that this solution was not realistic and that cooking should be included in the model, even though its uncertainty is large.

The solutions for Cases 1 through 4 were relatively stable with or without organics. Gasoline and diesel contributions were not resolved without organics. The overall burning contribution (hardwood plus softwood) depended mainly on $\mathrm{K}^{+}$ and not on organics. The cooking contribution was most influenced by $\mathrm{OC}$ and $\mathrm{OC} 3$, probably because cholesterol was lower than LQLs in most samples. However, when the 
Table 5. Modified pseudo-inverse normalized (MPIN) matrix in the CMB model for Case 1 of Table 4 . Key species for each source are underlined.

\begin{tabular}{|c|c|c|c|c|c|c|c|}
\hline $\begin{array}{l}\text { Species } \\
\text { code }^{\mathrm{a}}\end{array}$ & GAS & DIES & BURN-H & $\begin{array}{l}\text { Source code } \\
\text { BURN-S }\end{array}$ & COOK & AMSUL & AMNIT \\
\hline $\mathrm{NO}_{3}^{-}$ & 0.00 & 0.00 & 0.01 & -0.01 & 0.00 & -0.10 & 1.00 \\
\hline $\mathrm{SO}_{4}=$ & 0.00 & 0.00 & 0.00 & 0.00 & 0.00 & $\underline{1.00}$ & $-\overline{0.18}$ \\
\hline $\mathrm{NH}_{4}^{+}$ & 0.00 & 0.00 & -0.01 & 0.01 & 0.00 & $\overline{0.10}$ & 0.92 \\
\hline $\mathrm{Na}^{+}$ & -0.07 & -0.06 & 0.04 & 0.09 & 0.10 & 0.01 & 0.00 \\
\hline $\mathrm{K}^{+}$ & -0.04 & 0.00 & 1.00 & 0.00 & -0.30 & -0.06 & 0.00 \\
\hline OC3 & -0.04 & 0.02 & $\overline{0.10}$ & -0.20 & $\underline{0.52}$ & 0.00 & 0.00 \\
\hline OC4 & 0.00 & 0.04 & 0.12 & 0.00 & $\overline{0.14}$ & -0.02 & 0.00 \\
\hline OC & -0.07 & -0.03 & -0.01 & -0.10 & $\underline{1.00}$ & 0.00 & 0.00 \\
\hline $\mathrm{EC} 2$ & 0.06 & $\underline{1.00}$ & 0.37 & -0.64 & $-\overline{0.15}$ & -0.16 & 0.00 \\
\hline EC3 & 0.01 & 0.00 & -0.03 & 0.03 & 0.03 & 0.00 & 0.00 \\
\hline $\mathrm{EC}$ & -0.23 & 0.22 & -0.51 & $\underline{0.80}$ & -0.17 & -0.01 & 0.00 \\
\hline $\mathrm{Al}$ & -0.09 & -0.07 & -0.20 & $\overline{0.43}$ & -0.10 & 0.01 & 0.00 \\
\hline $\mathrm{Si}$ & $\underline{0.55}$ & -0.17 & -0.27 & 0.44 & -0.10 & -0.08 & -0.01 \\
\hline $\mathrm{Cl}$ & $\overline{0.03}$ & 0.02 & 0.21 & -0.12 & 0.01 & -0.02 & 0.00 \\
\hline K & -0.09 & -0.07 & $\underline{0.58}$ & 0.23 & -0.24 & -0.03 & 0.00 \\
\hline $\mathrm{Fe}$ & -0.19 & -0.12 & $-\overline{0.59}$ & 1.00 & -0.14 & 0.03 & 0.00 \\
\hline $\mathrm{Se}$ & 0.00 & 0.01 & 0.01 & $-\overline{0.01}$ & 0.00 & 0.00 & 0.00 \\
\hline $\mathrm{Br}$ & 0.13 & 0.15 & 0.12 & -0.16 & -0.03 & -0.05 & 0.00 \\
\hline $\mathrm{Pb}$ & 0.03 & -0.01 & -0.01 & -0.03 & 0.09 & 0.00 & 0.00 \\
\hline INCDPY & $\underline{0.54}$ & -0.14 & 0.00 & 0.06 & -0.05 & -0.08 & -0.01 \\
\hline BGHIPE & $\overline{0.93}$ & -0.16 & 0.09 & -0.16 & -0.03 & -0.14 & -0.01 \\
\hline CORONE & $\overline{1.00}$ & -0.14 & 0.13 & -0.23 & -0.06 & -0.15 & -0.01 \\
\hline HOP19 & $\underline{0.57}$ & -0.02 & 0.09 & -0.15 & -0.07 & -0.10 & -0.01 \\
\hline LEVGU & $\overline{0.05}$ & 0.06 & $\underline{0.50}$ & -0.25 & -0.08 & -0.04 & 0.00 \\
\hline SYRALD & 0.08 & 0.10 & $\underline{0.73}$ & -0.38 & -0.11 & -0.06 & 0.00 \\
\hline PALOL & -0.06 & 0.00 & $-\overline{0.06}$ & -0.19 & $\underline{0.49}$ & 0.02 & 0.00 \\
\hline OLAC & -0.02 & -0.01 & 0.00 & -0.08 & $\overline{0.20}$ & 0.01 & 0.00 \\
\hline CHOL & -0.03 & -0.02 & -0.05 & -0.07 & 0.22 & 0.01 & 0.00 \\
\hline NORFAR & 0.11 & 0.07 & 0.06 & -0.09 & -0.02 & -0.03 & 0.00 \\
\hline FARNES & 0.04 & 0.11 & 0.06 & -0.09 & -0.02 & -0.02 & 0.00 \\
\hline NORPRI & 0.04 & 0.23 & 0.10 & -0.20 & 0.02 & -0.04 & 0.00 \\
\hline PRISTU & -0.02 & 0.01 & -0.03 & -0.06 & 0.16 & 0.01 & 0.00 \\
\hline PHYTAN & -0.02 & 0.18 & 0.08 & -0.13 & -0.02 & -0.02 & 0.00 \\
\hline
\end{tabular}

a See Table 1 for chemical species.

cholesterol uncertainty was reduced to $10 \%$ of the average concentration, the solution remained similar to that of Case 1 , even though cholesterol became the most influential marker for cooking according to the MPIN. The cooking contribution is highly uncertain.

3.3 Source apportionment during winter (2000-2001) in Fresno

Each of the 51 samples collected in Fresno was subjected to CMB analysis. The average $\mathrm{r} 2$, chi-square, and percent mass accounted for were $0.89,1.78$, and $92 \%$, respectively, when organics were included in the CMB and 0.92, 1.23, and $104 \%$, respectively, without organics. Organics did not fit as well as traditional species, but including organics ac- counted for more of the measured mass. Table 6 presents average source contribution estimates (from CMB including organics) based on: 1) the duration-weighted average of the CMB results from the 51 individual samples (Case A); 2) the average of the $\mathrm{CMB}$ results from the four intensive periods (Case B); and 3) the CMB result of the duration-weighted average concentrations of the 51 individual samples (Case C). The species in Table 3 were included and CMB 8 was run in "auto fit" mode using the "s. elim." option to constrain the source contribution estimates to positive values.

In all cases, PVRD was not detected. GAS was larger than DIES in Cases A and B, although they were equivalent within stated uncertainty levels. The combined vehicle exhaust contributions were 14 and $15 \%$ of measured $\mathrm{PM}_{2.5}$. For Case C (average sample), DIES $\left(4.7 \mu \mathrm{g} / \mathrm{m}^{3}\right)$ was more 
Table 6. CMB source contribution estimates $\left(\mu \mathrm{g} / \mathrm{m}^{3}\right)$ for the CRPAQS winter intensive samples in Fresno.

\begin{tabular}{|c|c|c|c|c|c|c|}
\hline & $\begin{array}{c}\text { Case A } \\
\text { Average of } 51 \\
\text { individual samples }\end{array}$ & $\%^{\mathrm{a}}$ & $\begin{array}{c}\text { Case B } \\
\text { Average of } 4 \\
\text { IOP average samples }\end{array}$ & $\%$ & $\begin{array}{l}\text { Case C } \\
\text { Average } \\
\text { sample }\end{array}$ & $\%$ \\
\hline PVRD & $0.10 \pm 0.20$ & 0.1 & 0 & 0 & 0 & 0 \\
\hline GAS & $5.8 \pm 3.6$ & 8 & $6.9 \pm 4.0$ & 10 & $2.2 \pm 1.3$ & 3 \\
\hline DIES & $4.2 \pm 3.2$ & 6 & $3.6 \pm 3.9$ & 5 & $4.7 \pm 1.9$ & 6 \\
\hline MOBILE (sum) & $9.0 \pm 4.8$ & 14 & $10.5 \pm 5.6$ & 15 & $6.9 \pm 2.3$ & 9 \\
\hline BURN-H & $11.5 \pm 2.0$ & 16 & $11.7 \pm 2.5$ & 17 & $11.4 \pm 2.3$ & 16 \\
\hline BURN-S & $11.0 \pm 4.9$ & 15 & $8.7 \pm 7.2$ & 12 & $9.7 \pm 5.6$ & 13 \\
\hline BURN (sum) & $22 \pm 5$ & 31 & $20 \pm 8$ & 29 & $21 \pm 6$ & 29 \\
\hline COOK & $3.6 \pm 2.3$ & 5 & $7.9 \pm 3.3$ & 11 & $13.9 \pm 4.4$ & 19 \\
\hline AMSUL & $1.3 \pm 0.4$ & 2 & $1.2 \pm 0.3$ & 2 & $1.5 \pm 0.4$ & 2 \\
\hline AMNIT & $23 \pm 2$ & 32 & $22 \pm 2$ & 31 & $24 \pm 2$ & 33 \\
\hline MARINE & $0.09 \pm 0.09$ & 0.1 & $0.11 \pm 0.15$ & 0.2 & $0.08 \pm 0.22$ & 0.1 \\
\hline R SQR & 0.89 & & 0.94 & & 0.96 & \\
\hline CHI SQR & 1.8 & & 0.75 & & 0.67 & \\
\hline PCMASS $(\%)$ & 93 & & 91 & & 93 & \\
\hline Measured $\mathrm{PM}_{2.5}\left(\mu \mathrm{g} / \mathrm{m}^{3}\right)$ & 72 & & 70 & & 72 & \\
\hline
\end{tabular}

a Percent of measured $\mathrm{PM}_{2.5}$.

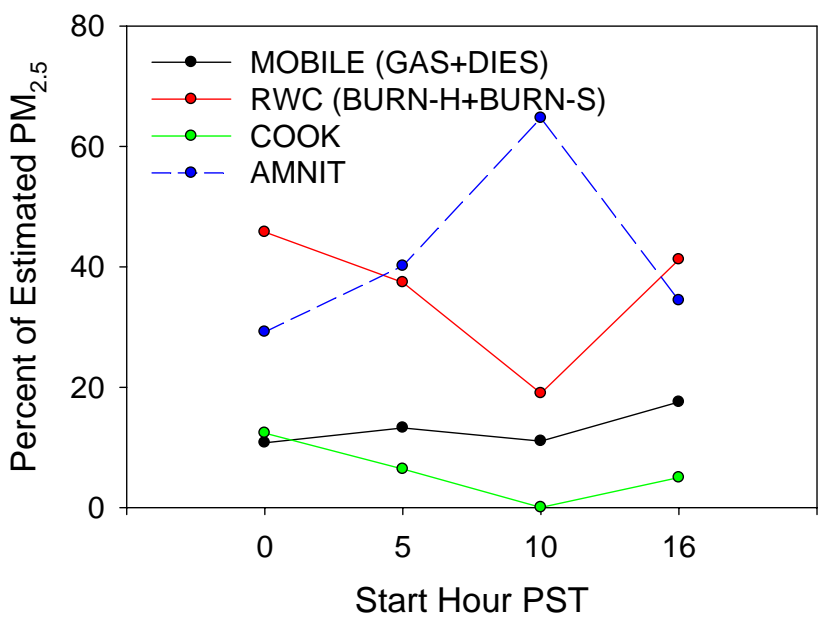

Fig. 1. Average diurnal variation of source contributions (percent of estimated $\mathrm{PM}_{2.5}$ ) for mobile (MOBILE = GAS + DIES), residential wood combustion (RWC $=$ BURN-H + BURN-S), cooking (COOK), and secondary ammonium nitrate (AMNIT) during the CRPAQS winter intensive study at the Fresno Supersite in California. The values represent averages from the four sample periods, 00:00-05:00, 05:00-10:00, 10:00-16:00, and 16:00-24:00 PST.

than twice GAS $\left(2.2 \mu \mathrm{g} / \mathrm{m}^{3}\right)$. The combined vehicle exhaust contribution was $9 \%$ of measured $\mathrm{PM}_{2.5}$. BURN-H was $16-17 \%$ in all cases, averaging $11.5 \mu \mathrm{g} / \mathrm{m}^{3}$. BURN-S ranged from $12-15 \%$ although its uncertainty was large, especially in Cases B and C. BURN-H and BURN-S combined ranged from $20 \mu \mathrm{g} / \mathrm{m}^{3}$ for Case B (29\%) to $22 \mu \mathrm{g} / \mathrm{m}^{3}$ for Case A $(31 \%)$. COOK was the most variable, ranging from
$3.6 \mu \mathrm{g} / \mathrm{m}^{3}$ (5\% of $\mathrm{PM}_{2.5}$ ) for Case A to $13.9 \mu \mathrm{g} / \mathrm{m}^{3}(19 \%$ of $\mathrm{PM}_{2.5}$ ) for Case C. AMSUL ranged from $1.2-1.5 \mu \mathrm{g} / \mathrm{m}^{3}$ ( $2 \%$ of PM 2.5 ), while AMNIT $\left(22-24 \mu \mathrm{g} / \mathrm{m}^{3}\right)$, accounted for $31-33 \%$ of $\mathrm{PM}_{2.5}$. The MARINE contribution was not significant in any of the cases. Overall, $\mathrm{PM}_{2.5}$ mass was underestimated by less than $10 \%$. The CMB performance measures were better for average samples (Cases B and C) than for individual samples (Case A).

Average diurnal variations of source contributions are presented in Fig. 1. Average source contributions derived from CMB analysis, including organics, from mobile (MOBILE $=$ GAS + DEISEL), residential wood combustion $($ RWC $=$ BURN-H + BURN-S), cooking (COOK), and secondary ammonium nitrate (AMNIT) for the 00:00-05:00, 05:00-10:00, 10:00-16:00, and 16:00-24:00 PST periods were calculated as a percentage of total estimated $\mathrm{PM}_{2.5}$ mass. AMNIT increased in the afternoon period (10:00-16:00 PST) as transported pollutants were mixed to the surface (Watson and Chow, 2002; Chow et al., 2006a). Cooking and burning contributions displayed similar diurnal variations, with the highest relative contributions in the evening (16:00-24:00 PST) and early morning hours (00:00-05:00 PST). The mobile contribution varied least during the day although the percent contributions were highest in the evening and mid-morning (05:00-10:00) periods. Watson et al. (2002b, 2006b) drew similar conclusions about diurnal variations of source contributions in Fresno from continuous measurements of particle size distributions and $\mathrm{NO}_{\mathrm{x}}, \mathrm{CO}$, and black carbon concentrations. 


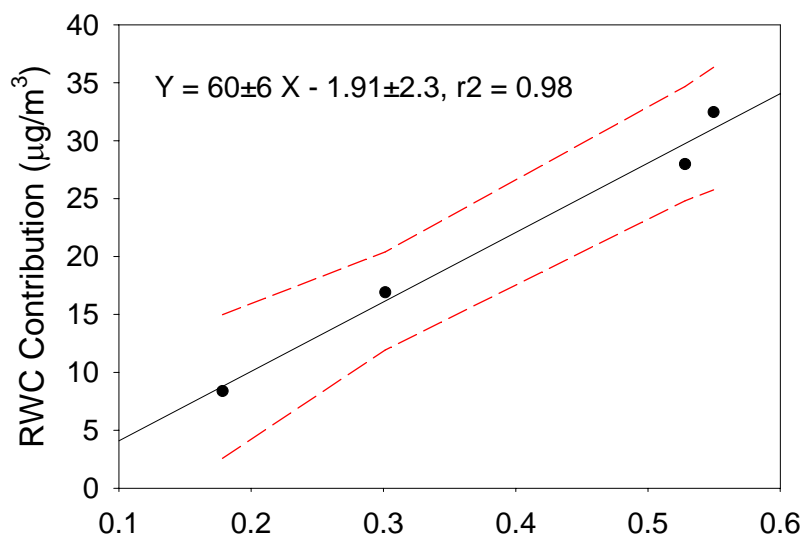

Ambient Water-Soluble K $\left(\mu \mathrm{g} / \mathrm{m}^{3}\right)$

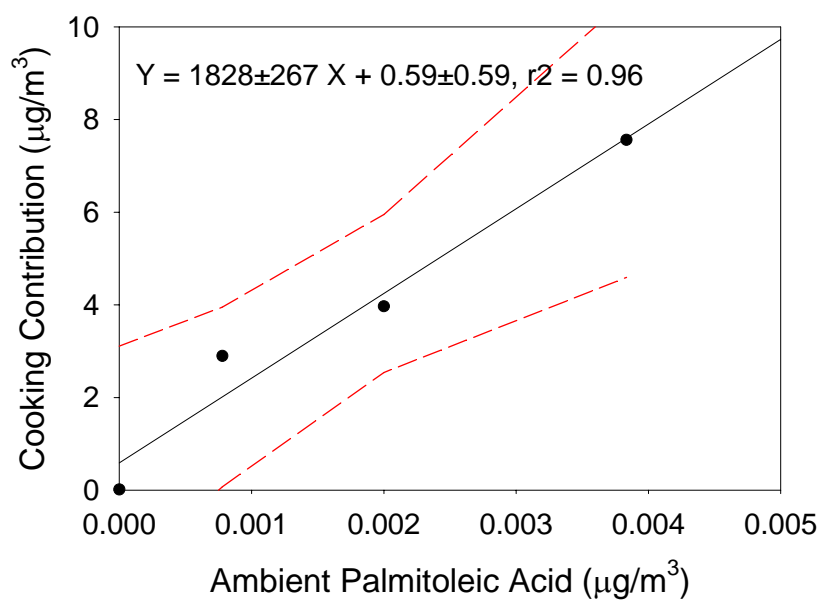

Fig. 2. Comparison of average residential wood combustion (RWC) and cooking contributions and average ambient water-soluble potassium $\left(\mathrm{K}^{+}\right)$and palmitoleic acid concentrations during four CRPAQS winter intensive periods at the Fresno Supersite in California. The values represent averages from the four sample periods during the winter intensive study (00:00-05:00, 05:00-10:00, 10:0016:00, and 16:00-24:00 PST). Included in the figure are the regression parameters and the $95 \%$ confidence interval of the expected values of the dependent variable.

While deviations between the measured source profiles and the composition of actual emissions near the Fresno Supersite are probably the largest source of uncertainty, it is difficult to assess the magnitude of these errors. Applying the source profiles to simulated data defines expected estimation error under ideal conditions where such errors are random. $\mathrm{CMB}$ analysis of ambient concentrations averaged on various time scales provides bounds on source contribution estimates under real-world conditions. Reported cholesterol and palmitoleic acid concentrations were larger than their measurement uncertainties for only 12 and $25 \%$, respectively, of the Fresno samples. The inability to detect cooking markers probably contributed to large uncertainties for the estimated
Table 7. Fresno source contributions (\%) from CMB during IMS95 (Schauer and Cass, 2000) and CRPAQS winter intensive study. Also shown are contributions from the California emission inventory (CARB, 2004).

\begin{tabular}{lccc}
\hline Source & IMS95 $^{\mathrm{a}}$ & CRPAQS $^{\mathrm{b}}$ & $\begin{array}{c}\text { SJV Emission }^{\mathrm{c}} \\
\text { Inventory }^{\mathrm{c}}\end{array}$ \\
\hline Paved road dust & 0 & 0 & 22 \\
Vehicle exhaust (gasoline) & 3 & 7 & - \\
Vehicle exhaust (diesel) & 10 & 6 & - \\
Vehicle exhaust (combined) & 13 & 13 & 8 \\
Wood burning & 41 & 30 & 11 \\
Cooking & 8 & 12 & 2 \\
Secondary ammonium sulfate & 4 & 2 & - \\
Secondary ammonium nitrate & 30 & 32 & - \\
Marine & - & 0 & - \\
\hline
\end{tabular}

a Percent of estimated $\mathrm{PM}_{2.5}$ mass.

$\mathrm{b}$ Percent of measured $\mathrm{PM}_{2.5}$ mass.

c Renormalized to include secondary ammonium sulfate and ammonium nitrate.

cooking contribution, i.e., from 5 to $19 \%$ of $\mathrm{PM}_{2.5}$. On the other hand, the total RWC contribution was stable.

Figure 2 shows the relationships between measured $\mathrm{K}^{+}$ concentrations and RWC contributions as well as between palmitoleic acid concentrations and cooking contributions. The data were averaged because most of the palmitoleic concentrations in the individual samples were reported as zero. There were 13, 13, 12, and 13 samples included in the averages for the 00:00-05:00, 05:00-10:00, 10:00-16:00, and 16:00-24:00 PST periods, respectively. There are clear relationships between the wood smoke and cooking markers $\left(\mathrm{K}^{+}\right.$ and palmitoleic acid, respectively) and the corresponding estimated source contributions. These relationships are insufficient to guarantee that the source contribution estimates are unbiased unless the compositions of the marker species in the source profiles are realistic.

Table 7 compares the average source contributions (\%) from Cases A-C in Table 6 with the 1995 Fresno source apportionments reported by Schauer and Cass (2000). In general, the fractions contributed by each source type are similar, although this study estimates slightly higher gasolinethan diesel-exhaust contributions. Schauer and Cass (2000) estimated $37 \%$ higher wood burning and this study estimates $50 \%$ higher cooking contributions. These differences result from a combination of the different measurement and modeling methods, as well as possible differences in the actual source contributions. In both cases, wood burning dominates the OC contributions.

Also shown in Table 7 are source contributions taken from the California emission inventory (California Air Resources Board, 2004), described above. Because the inventory represents primary $\mathrm{PM}_{2.5}$ emissions, these values were renormalized to include the secondary $\left(\mathrm{NH}_{4}\right)_{2} \mathrm{SO}_{4}$ and $\mathrm{NH}_{4} \mathrm{NO}_{3}$ contributions. The biggest difference between the inventory 
and these results is the high fugitive dust fraction (22\%) in the inventory. The inventory represents all of California for the entire year, and rural agricultural areas may experience higher fugitive dust impacts during drier, non-winter periods (e.g., Chow et al., 2006a). While the CMB (13\%) and inventory-based ( $8 \%$ ) vehicle contributions were similar, the wood burning and cooking contributions in the inventory (11 and $2 \%$, respectively) were much lower than those estimated by CMB (36 and 10\%, respectively). Again, these differences may be related in part to real geographical and seasonal variability in the source impacts.

\section{Conclusions}

Including organic compounds in the CMB improved the distinction between gasoline and diesel vehicle emissions and allowed a more precise estimate of the cooking source contribution. However, organics were not required to precisely estimate the RWC contribution and did not increase the precision of the softwood burning contribution even though there were significant differences in the hardwood and softwood compositions of RWC markers such as levoglucosan, 4-allylguaiacol, and syringaldehyde. The most important RWC marker in the Fresno CMB analysis was water-soluble $\mathrm{K}^{+}$, but this was not sufficient to distinguish between hardwood and softwood combustion.

RWC was the largest contributor to measured $\mathrm{PM}_{2.5}$ (29$31 \%$ ). Hardwood and softwood combustion accounted for $16-17 \%$ and $12-15 \%$ of $\mathrm{PM}_{2.5}$, respectively, although the uncertainty of the softwood contribution was large. Secondary $\mathrm{NH}_{4} \mathrm{NO}_{3}$ represented $31-33 \%$ of $\mathrm{PM}_{2.5}$. Motor vehicle exhaust contributed only $9-15 \%$ of $\mathrm{PM}_{2.5}$. The gasolinevehicle contribution (3-10\%) was comparable to the dieselvehicle contribution (5-6\%). The cooking contribution did not depend on cholesterol, which was not detected in most samples, and was uncertain, ranging from 5-19\% of $\mathrm{PM}_{2.5}$. The most important markers for cooking were OC (specifically $\mathrm{OC} 3$, the carbon fraction evolved at $450^{\circ} \mathrm{C}$ in an inert atmosphere) and palmitoleic acid. However, cholesterol and palmitoleic acid are not unique to meat cooking and more research is needed to identify other markers in the cooking source profiles. Improved sampling and analytic approaches are also needed to accurately measure these species on the short time scales $(5-8 \mathrm{~h})$. Despite this variability, this analysis suggests that cooking was an important $\mathrm{PM}_{2.5}$ contributor at Fresno. The current Fresno source contribution estimates are consistent with 1995 receptor modeling using organic markers (Schauer and Cass, 2000).

Acknowledgements. We would like to thank E. Fujita and D. Campbell for providing the Gas/Diesel Split motor vehicle source profiles. The Fresno Supersite is a cooperative effort between the California Air Resources Board (ARB) and the Desert Research Institute (DRI). Sponsorship is provided by the U.S. Environmental Protection Agency Contract \#R-82805701. This work was also supported by the California Regional $\mathrm{PM}_{10} / \mathrm{PM}_{2.5}$ Air Quality Study (CRPAQS) Agency under the management of the California Air Resources Board and by the U.S. Environmental Protection Agency under STAR Grant \#RD-83108601-0. Any mention of commercially available products and supplies does not constitute an endorsement of those products and supplies.

Edited by: M. Ammann

\section{References}

Ashbaugh, L. L., Carvacho, O. F., Brown, M. S., Chow, J. C., Watson, J. G., and Magliano, K. L.: Soil sample collection and analysis for the Fugitive Dust Characterization Study, Atmos. Environ., 37(9-10), 1163-1173, 2003.

California Air Resources Board: Climate Change Emissions Inventory, Draft Report, prepared by California Environmental Protection Agency Air Resources Board, Sacramento, CA, 2004.

Chen, L.-W. A., Chow, J. C., Watson, J. G., Lowenthal, D. H., and Chang, M.-C. O.: Quantifying $\mathrm{PM}_{2.5}$ source contributions for the San Joaquin Valley with multivariate receptor models, Environ. Sci. Technol., accepted, 2007.

Chow, J. C., Watson, J. G., Lowenthal, D. H., Solomon, P. A., Magliano, K. L., Ziman, S. D., and Richards, L. W.: PM 10 source apportionment in California's San Joaquin Valley, Atmos. Environ., 26A(18), 3335-3354, 1992.

Chow, J. C., Watson, J. G., Pritchett, L. C., Pierson, W. R., Frazier, C. A., and Purcell, R. G.: The DRI Thermal/Optical Reflectance carbon analysis system: Description, evaluation and applications in U.S. air quality studies, Atmos. Environ., 27A(8), 1185-1201, 1993.

Chow, J. C.: Critical review: Measurement methods to determine compliance with ambient air quality standards for suspended particles. J. Air Waste Manage. Assoc., 45, 320-382. 1995.

Chow, J. C., Watson, J. G., Lowenthal, D. H., and Countess, R. J.: Sources and chemistry of $\mathrm{PM}_{10}$ aerosol in Santa Barbara County, CA, Atmos. Environ., 30(9), 1489-1499, 1996.

Chow, J. C. and Watson, J. G.: Ion chromatography in elemental analysis of airborne particles, in: Elemental Analysis of Airborne Particles, vol. 1, edited by: Landsberger, S. and Creatchman, M., Gordon and Breach Science, Amsterdam, 97-137, 1999.

Chow, J. C., Watson, J. G., Crow, D., Lowenthal, D. H., and Merrifield, T. M.: Comparison of IMPROVE and NIOSH carbon measurements, Aerosol Sci. Technol., 34(1), 23-34, 2001.

Chow, J. C. and Watson, J. G.: $\mathrm{PM}_{2.5}$ carbonate concentrations at regionally representative Interagency Monitoring of Protected Visual Environment sites, J. Geophys. Res., 107(D21), ICC 6-1ICC 6-9, 2002.

Chow, J. C., Watson, J. G., Ashbaugh, L. L., and Magliano, K. L.: Similarities and differences in $\mathrm{PM}_{10}$ chemical source profiles for geological dust from the San Joaquin Valley, California, Atmos. Environ., 37(9-10), 1317-1340, 2003.

Chow, J. C., Watson, J. G., Chen, L.-W. A., Arnott, W. P., Moosmüller, H., and Fung, K. K.: Equivalence of elemental carbon by Thermal/Optical Reflectance and Transmittance with different temperature protocols, Environ. Sci. Technol., 38(16), 4414-4422, 2004a.

Chow, J. C., Watson, J. G., Kuhns, H. D., Etyemezian, V., Lowenthal, D. H., Crow, D. J., Kohl, S. D., Engelbrecht, J. P., and 
Green, M. C.: Source profiles for industrial, mobile, and area sources in the Big Bend Regional Aerosol Visibility and Observational (BRAVO) Study, Chemosphere, 54(2), 185-208, $2004 \mathrm{~b}$.

Chow, J. C., Chen, L.-W. A., Lowenthal, D. H., Doraiswamy, P., Park, K., Kohl, S., Trimble, D. L., and Watson, J. G.: California Regional $\mathrm{PM}_{10} / \mathrm{PM}_{2.5}$ Air Quality Study (CRPAQS) - Initial data analysis of field program measurements, prepared for California Air Resources Board, Sacramento, CA by Desert Research Institute, Reno, NV, 2005a.

Chow, J. C., Watson, J. G., Lowenthal, D. H., and Magliano, K. L.: Loss of $\mathrm{PM}_{2.5}$ nitrate from filter samples in central California, J. Air Waste Manage. Assoc., 55(8), 1158-1168, 2005 b.

Chow, J. C., Watson, J. G., Chen, L. W. A., Paredes-Miranda, G., Chang, M.-C. O., Trimble, D., Fung, K. K., Zhang, H., and Yu, J. Z.: Refining temperature measures in thermal/optical carbon analysis, Atmos. Chem. Phys., 5, 2961-2972, 2005c.

Chow, J. C., Chen, L.-W. A., Watson, J. G., Lowenthal, D. H., Magliano, K. L., Turkiewicz, K., and Lehrman, D.: PM 2.5 chemical composition and spatiotemporal variability during the California Regional $\mathrm{PM}_{10} / \mathrm{PM}_{2.5}$ Air Quality Study (CRPAQS), J. Geophys. Res., 111(D10), D10S04, doi:10.1029/2005JD006457, 2006a.

Chow, J. C., Watson, J. G., Lowenthal, D. H., Chen, L. W. A., and Magliano, K. L.: Particulate carbon measurements in California's San Joaquin Valley, Chemosphere, 62(3), 337-348, $2006 \mathrm{~b}$.

Dreyfus, M. A., Tolocka, M. P., Dodds, S. M., Dykins, J., and Johnston, M. V.: Cholesterol ozonolysis: Kinetics, mechanism and oligomer products, J. Phys. Chem. A, 109, 6242-6248, 2005.

Fitz, D. R., Chow, J. C., and Zielinska, B.: Development of a gas and particulate matter organic speciation profile database, prepared for Draft Final Report June 2003, Prepared fro San Joaquin Valleywide Air Pollution Study Agency; California Regional $\mathrm{PM}_{10} / \mathrm{PM}_{2.5}$ Air Quality Study by Desert Research Institute, Reno, NV, 2003.

Fraser, M. P., Cass, G. R., and Simoneit, B. R. T.: Air quality model evaluation data for organics 6. C3-C24 organic acids, Environ. Sci. Technol., 37(3), 446-453, 2003.

Fujita, E. M., Zielinska, B., Arnott, W. P., Campbell, D. E., Reinhart, L., Sagebiel, J. C., and Chow, J. C.: Gasoline/Diesel PM Split Study: Source and ambient sampling, chemical analysis, and apportionment phase, final report, prepared for National Renewable Energy Laboratory, Golden, CO by Desert Research Institute, Reno, NV, 2006.

Hannigan, M. P., Busby Jr., W. F., and Cass, G. R.: Source contributions to the mutagenicity of urban particulate air pollution, J. Air Waste Manage. Assoc., 55(4), 399-410, 2005.

Hidy, G. M. and Friedlander, S. K.: The nature of the Los Angeles aerosol, in: Proceedings of the Second International Clean Air Congress, edited by: Englund, H. M. and Beery, W. T., 391-404, 1971.

Hoffman, G. L. and Duce, R. A.: Copper contamination of atmospheric particulate samples collected with Gelman hurricane air sampler, Environ. Sci. Technol., 5, 1134-1136, 1971.

Javitz, H. S., Watson, J. G., and Robinson, N. F.: Performance of the chemical mass balance model with simulated local-scale aerosols, Atmos. Environ., 22, 10, 2309-2322, 1988.

Kim, B. M. and Henry, R. C.: Diagnostics for determining influential species in the chemical mass balance receptor model, J. Air Waste Manage. Assoc., 49(12), 1449-1455, 1999.
King, R. B. and Toma, J.: Copper emissions from a high-volume air sampler, NASA Technical Memorandum, 1975.

Labban, R., Veranth, J. M., Watson, J. G., and Chow, J. C.: Feasibility of soil dust source apportionment by pyrolysis-gas chromatography/mass spectrometry method, J. Air Waste Manage. Assoc., 56(9), 1230-1242. 2006.

Lowenthal, D. H., Chow, J. C., Watson, J. G., Neuroth, G. R., Robbins, R. B., Shafritz, B. P., and Countess, R. J.: The effects of collinearity on the ability to determine aerosol contributions from diesel- and gasoline-powered vehicles using the chemical mass balance model, Atmos. Environ., 26A(13), 2341-2351, 1992.

Magliano, K. L., Hughes, V. M., Chinkin, L. R., Coe, D. L., Haste, T. L., Kumar, N., and Lurmann, F. W.: Spatial and temporal variations in $\mathrm{PM}_{10}$ and $\mathrm{PM}_{2.5}$ source contributions and comparison to emissions during the 1995 Integrated Monitoring Study, Atmos. Environ., 33(29), 4757-4773, 1999.

Mamane, Y. and Gottlieb, J.: Nitrate formation on sea-salt and mineral particles - A single particle approach, Atmos. Environ., 26A(9), 1763-1769, 1992.

Manchester-Neesvig, J. B., Schauer, J. J., and Cass, G. R.: The distribution of particle-phase organic compounds in the atmosphere and their use for source apportionment during the Southern California Children's Health Study, J. Air Waste Manage. Assoc., 53(9), 1065-1079, 2003.

McDonald, J. D., Zielinska, B., Fujita, E. M., Sagebiel, J. C., Chow, J. C., and Watson, J. G.: Emissions from charbroiling and grilling of chicken and beef, J. Air Waste Manage. Assoc., 53(2), 185194, 2003.

McDonald, J. D., Zielinska, B., Fujita, E. M., Sagebiel, J. C., Chow, J. C., and Watson, J. G.: Fine particle and gaseous emission rates from residential wood combustion, Environ. Sci. Technol., 34(11), 2080-2091, 2000.

Miguel, A. H., Kirchstetter, T. W., Harley, R. A., and Hering, S. V.: On-road emissions of particulate polycyclic aromatic hydrocarbons and black carbon soot from gasoline and diesel vehicles, Enivron. Sci. Technol., 32(4), 450-455, 1998.

Park, K., Chow, J. C., Watson, J. G., Trimble, D. L., Doraiswamy, P., Arnott, W. P., Stroud, K. R., Bowers, K., Bode, R., Petzold, A., and Hansen, A. D. A.: Comparison of continuous and filterbased carbon measurements at the Fresno Supersite, J. Air Waste Manage. Assoc., 56(4), 474-491, 2006.

Patterson, R. K.: Aerosol contamination from high volume sampler exhaust, J. Air Poll. Control Assoc., 30(2), 169-171, 1980.

Rinehart, L. R.: The origin of polar organic compounds in ambient fine particulate matter, Ph.D. Dissertation, University of Nevada, Reno, 2005.

Rinehart, L. R., Fujita, E. M., Chow, J. C., Magliano, K. L., and Zielinska, B.: Spatial distribution of $\mathrm{PM}_{2.5}$ associated organic compounds in central California, Atmos. Environ., 40(2), 290303, 2006.

Rogge, W. F., Hildemann, L. M., Mazurek, M. A., Cass, G. R., and Simoneit, B. R. T.: Sources of fine organic aerosol - 1. Charbroilers and meat cooking operations, Enivron. Sci. Technol., 25(6), 1112-1125, 1991.

Schauer, J. J. and Cass, G. R.: Source apportionment of wintertime gas-phase and particle-phase air pollutants using organic compounds as tracers, Environ. Sci. Technol., 34(9), 1821-1832, 2000.

Schauer, J. J., Rogge, W. F., Mazurek, M. A., Hildemann, L. M., 
Cass, G. R., and Simoneit, B. R. T.: Source apportionment of airborne particulate matter using organic compounds as tracers, Atmos. Environ., 30(22), 3837-3855, 1996.

Simoneit, B. R. T.: Organic matter of the troposphere - V: Application of molecular marker analysis to vehicular exhaust for source reconciliations, Int. J. Environ. Anal. Chem., 22, 203-233, 1985.

Turpin, B. J., Huntzicker, J. J., and Hering, S. V.: Investigation of organic aerosol sampling artifacts in the Los Angeles Basin, Atmos. Environ., 28, 19, 3061-3071, 1994.

U.S. EPA: SPECIATE database, http://www.epa.gov/ttn/chief/ software/speciate/index.html, last accessed 13 February 2007.

Wang, D. and Hopke, P. K.: The use of constrained least-squares to solve the chemical mass balance problem, Atmos. Environ., 23(10), 2143-2150, 1989.

Watson, J. G., Cooper, J. A., and Huntzicker, J. J.: The effective variance weighting for least squares calculations applied to the mass balance receptor model, Atmos. Environ., 18(7), 1347$1355,1984$.

Watson, J. G., Chow, J. C., Lowenthal, D. H., Pritchett, L. C., Frazier, C. A., Neuroth, G. R., and Robbins, R.: Differences in the carbon composition of source profiles for diesel- and gasolinepowered vehicles, Atmos. Environ., 28(15), 2493-2505, 1994.

Watson, J. G., Robinson, N. F., Lewis, C. W., Coulter, C. T., Chow, J. C., Fujita, E. M., Lowenthal, D. H., Conner, T. L., Henry, R. C., and Willis, R. D.: Chemical mass balance receptor model version 8 (CMB) user's manual, prepared for U.S. Environmental Protection Agency, Research Triangle Park, NC by Desert Research Institute, Reno, NV, 1997.

Watson, J. G., DuBois, D. W., DeMandel, R., Kaduwela, A. P., Magliano, K. L., McDade, C., Mueller, P. K., Ranzieri, A. J., Roth, P. M., and Tanrikulu, S.: Field program plan for the California Regional $\mathrm{PM}_{2.5} / \mathrm{PM}_{10}$ Air Quality Study (CRPAQS), prepared for California Air Resources Board, Sacramento, CA by Desert Research Institute, Reno, NV, 1998a.

Watson, J. G., Robinson, N. F., Lewis, C. W., Coulter, C. T., Chow, J. C., Fujita, E. M., Conner, T. L., and Pace, T. G.: CMB8 applications and validation protocol for $\mathrm{PM}_{2.5}$ and VOCs, prepared for U.S. Environmental Protection Agency, Research Triangle Park, NC by Desert Research Institute, Reno, NV, 1998b.

Watson, J. G., Chow, J. C., and Frazier, C. A.: X-ray fluorescence analysis of ambient air samples, in: Elemental Analysis of Airborne Particles, vol. 1, edited by: Landsberger, S. and Creatchman, M., Gordon and Breach Science, Amsterdam, 67-96, 1999.

Watson, J. G., Chow, J. C., Bowen, J. L., Lowenthal, D. H., Hering, S., Ouchida, P., and Oslund, W.: Air quality measurements from the Fresno Supersite, J. Air Waste Manage. Assoc., 50(8), 13211334, 2000.

Watson, J. G. and Chow, J. C.: A wintertime $\mathrm{PM}_{2.5}$ episode at the Fresno, CA, supersite, Atmos. Environ., 36(3), 465-475, 2002.

Watson, J. G., Zhu, T., Chow, J. C., Engelbrecht, J. P., Fujita, E. M., and Wilson, W. E.: Receptor modeling application framework for particle source apportionment, Chemosphere, 49(9), 1093-1136, 2002a.
Watson, J. G., Chow, J. C., Lowenthal, D. H., Stolzenburg, M. R., Kreisberg, N. M., and Hering, S. V.: Particle size relationships at the Fresno supersite, J. Air Waste Manage. Assoc., 52(7), 822827,2002 b.

Watson, J. G. and Chow, J. C.: Receptor models for air quality management, EM, 10, October, 27-36, 2004.

Watson, J. G. and Chow, J. C.: Receptor models, in: Air Quality Modeling - Theories, Methodologies, Computational Techniques, and Available Databases and Software, vol. II - Advanced Topics, edited by: Zannetti, P., Air Waste Manage. Assoc. and the EnviroComp Institute, Pittsburgh, PA, 455-501, 2005.

Watson, J. G., Chow, J. C., and Chen, L.-W. A.: Summary of organic and elemental carbon/black carbon analysis methods and intercomparisons, Aerosol Air Quality Res., 5(1), 65-102, 2005.

Watson, J. G., Chow, J. C., Lowenthal, D. H., Kreisberg, N., Hering, S. V., and Stolzenburg, M. R.: Variations of nanoparticle concentrations at the Fresno supersite, Sci. Total Environ., 358(1-3), 178-187, 2006a.

Watson, J. G., Chow, J. C., Park, K., and Lowenthal, D. H.: Nanoparticle and ultrafine particle events at the Fresno Supersite, J. Air Waste Manage. Assoc., 56(4), 417-430, $2006 \mathrm{~b}$.

Zheng, M., Cass, G. R., Schauer, J. J., and Edgerton, E. S.: Source apportionment of $\mathrm{PM}_{2.5}$ in the southeastern United States using solvent-extractable organic compounds as tracers, Environ. Sci. Technol., 36(11), 2361-2371, 2002.

Zheng, M., Ke, L., Edgerton, E. S., Schauer, J. J., Dong, M. Y., and Russell, A. G.: Spatial distribution of carbonaceous aerosol in the southeastern United States using molecular markers and carbon isotope data, J. Geophys. Res., 111(D10), D10S06, doi:10.1029/2005JD006777, 2006.

Zielinska, B., McDonald, J. D., Hayes, T., Chow, J. C., Fujita, E. M., and Watson, J. G.: Northern Front Range Air Quality Study, Volume B: Source measurements, prepared for Colorado State University, Fort Collins, CO by Desert Research Institute, Reno, NV, 1998.

Zielinska, B. and Fujita, E. M.: Characterization of ambient volatile organic compounds at the western boundary of the SCOS97NARSTO modeling domain, Atmos. Environ., 37, Suppl. 2, S171-S180, 2003.

Zielinska, B., Rinehart, L. R., and Goliff, W. S.: California Regional $\mathrm{PM}_{10} / \mathrm{PM}_{2.5}$ Air Quality Study - Organic compound measurements, prepared for California Air Resources Board, Sacramento, CA by Desert Research Institute, Reno, NV, 2003.

Zielinska, B., Sagebiel, J., Whitney, K., and Lawson, D. R.: Emission rates and comparative chemical composition from selected in-use diesel and gasoline-fueled vehicles, J. Air Waste Manage. Assoc., 54(9), 1138-1150, 2004a.

Zielinska, B., Sagebiel, J., Arnott, W. P., Rogers, C. F., Kelly, K. E., Wagner, D. A., Lighty, J. S., Sarofim, A. F., and Palmer, G.: Phase and size distribution of polycyclic aromatic hydrocarbons in diesel and gasoline vehicle emissions, Environ. Sci. Technol., 38(9), 2557-2567, 2004b. 